\title{
A Multimodal MR Imaging Study of the Effect of Hippocampal Damage on Affective and Cognitive Functions in a Rat Model of Chronic Exposure to a Plateau Environment
}

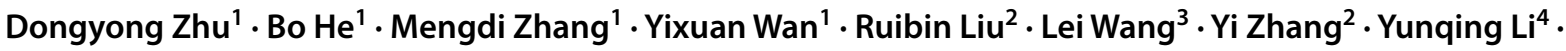 \\ Fabao Gao ${ }^{1,3}$
}

Received: 6 September 2021 / Revised: 18 November 2021 / Accepted: 23 November 2021 / Published online: 4 January 2022

(c) The Author(s) 2021

\begin{abstract}
Prolonged exposure to high altitudes above $2500 \mathrm{~m}$ above sea level (a.s.l.) can cause cognitive and behavioral dysfunctions. Herein, we sought to investigate the effects of chronic exposure to plateau hypoxia on the hippocampus in a rat model by using voxel-based morphometry, creatine chemical exchange saturation transfer (CrCEST) and dynamic contrast-enhanced MR imaging techniques. 58 healthy 4-week-old male rats were randomized into plateau hypoxia rats ( $\mathrm{H}$ group) as the experimental group and plain rats (P group) as the control group. H group rats were transported from Chengdu (500 $\mathrm{m}$ a.s.1.), a city in a plateau located in southwestern China, to the Qinghai-Tibet Plateau (4250 m a.s.l.), Yushu, China, and then fed for 8 months there, while $\mathrm{P}$ group rats were fed in Chengdu (500 m a.s.1.), China. After 8 months of exposure to plateau hypoxia, open-field and elevated plus maze tests revealed that the anxiety-like behavior of the $\mathrm{H}$ group rats was more serious than that of the $\mathrm{P}$ group rats, and the Morris water maze test revealed impaired spatial memory function in the $\mathrm{H}$ group rats. Multimodal MR imaging analysis revealed a decreased volume of the regional gray matter, lower CrCEST contrast and higher transport coefficient Ktrans in the hippocampus compared with the $\mathrm{P}$ group rats. Further correlation analysis found associations of quantitative MRI parameters of the hippocampus with the behavioral performance of $\mathrm{H}$ group rats. In this study, we validated the viability of using noninvasive multimodal MR imaging techniques to evaluate the effects of chronic exposure to a plateau hypoxic environment on the hippocampus.
\end{abstract}

Keywords Voxel-based morphometry · Chemical exchange saturation transfer · Dynamic contrast-enhanced MR imaging · Animal model $\cdot$ Hippocampal damage $\cdot$ Plateau hypoxia

Fabao Gao

gaofabao@wchscu.cn

1 Department of Radiology, West China Hospital, Sichuan University, No. 37 Guoxue Road, Chengdu 610041, China

2 Department of Biomedical Engineering, College of Biomedical Engineering and Instrument Science, Zhejiang University, Hangzhou 310030, China

3 Molecular Imaging Center, West China Hospital, Sichuan University, Chengdu 610041, China

4 Department of Anatomy and KK Leung Brain Research Centre, The Fourth Military Medical University, Xi' an 710032, China

\section{Introduction}

Hypobaric hypoxic exposure is a hazard that can lead to severe abnormalities in physiological and psychological functions due to strong stress responses of the body. Most of the previous studies established high-altitude hypoxic animal models using an animal decompression chamber. Simulated hypobaric hypoxia is often needed to bring the chamber down to sea level to replenish food and water at regular intervals, which is apparently different from genuine plateau environments. In this study, we established a rat brain damage model under chronic exposure to a natural plateau hypoxic environment. It has been widely reported that prolonged exposure to high-altitude hypobaric hypoxia may induce deficits in learning and memory [1-3]. As the hippocampus is closely associated with explicit memory, especially spatial learning and memory, any insult to this 
region can affect cognitive functions [4]. Studies of the differential susceptibility of brain regions to hypobaric hypoxia suggest that the hippocampus is more susceptible to hypoxic stress than the cerebellum, the cortex and the striatum $[5,6]$. Hypobaric hypoxia-induced stress can cause stress in hippocampal oxidation, synaptic dysfunction, neurodegeneration and apoptosis and thus result in learning and memory deficits [7-10]. Therefore, it is necessary to research and develop effective and widely available biomarkers for the early detection of hippocampal damage.

Among the many techniques applicable to such early detection are multimodal MR imaging techniques [11], such as voxel-based morphometry (VBM), chemical exchange saturation transfer (CEST) imaging and dynamic contrastenhanced MR imaging (DCE-MRI). They provide noninvasive means to detect morphological alterations in the gray matter, macromolecular protein level and cerebrovascular microcirculation. VBM, based on voxel differences in tissue classification, provides an effective neuroimaging approach to the investigation of subtle and discrete changes in regional gray matter volume (rGMV)/density in the entire brain [12]. A previous neuroimaging study [13] of high-altitude sojourners revealed significant gray matter loss and atrophy in several cerebral regions, including the cortex, hippocampus and striatum, suggesting that hypobaric hypoxia exposure may result in neuronal damage and impair cognitive functions [14]. However, to date, it has remained unknown whether VBM-detected changes in the regional gray matter of the hippocampus are directly linked to memory functions and mood.

In addition to VBM is CEST imaging, which has also been reported to be sensitive to macromolecular metabolites and proteins in tissues $[15,16]$. Creatine CEST (CrCEST) contrast, enabling $\mathrm{Cr}$ imaging with high sensitivity and good spatial resolution [17], has been used as an in vivo tool for measuring free $\mathrm{Cr}$ because it can visualize and quantify oxidative phosphorylation capacity [18]. Hypobaric hypoxia exposure may obstruct the energy metabolic pathway and result in mitochondrial dysfunction and energy disorders [19], thus damaging and even killing cells in the brain [20]. In the present study, we sought to investigate the feasibility of the CrCEST for investigating changes in $\mathrm{Cr}$ in the brain and to evaluate the effect of hippocampal damage on affective and cognitive behaviors in a rat model of chronic exposure to hypobaric hypoxia.

DCE-MRI, the third multimodal MRI technique for the early detection of hippocampal damage, can quantify permeability in vivo even in small animals by assessing the diffusive transport of gadopentetate dimeglumine across the blood-brain barrier (BBB) [21, 22]. Previous studies have reported that chronic exposure to hypobaric hypoxia can biochemically alter the BBB [23] and increase its permeability [24]. To fill this niche in DCE-MRI, it is necessary to explore the influence of chronic exposure to hypobaric hypoxia on cerebral microvessels by using DCE-MRI to assess BBB permeability.

To the best of our knowledge, few studies have integrated the VBM, CrCEST and DCE-MRI techniques to evaluate the effects of plateau hypoxia exposure on the brain in a comprehensive manner. It is under such circumstances and to fill the abovementioned niches that this study is conducted to use the above three multimodal MR imaging techniques to detect alterations in $\mathrm{rGMV}$, free $\mathrm{Cr}$ concentration, and $\mathrm{BBB}$ function of the brain in a rat model chronically exposed to a plateau hypoxic environment and thus to verify whether the regional changes in the brain could reveal the mechanisms underlying hypobaric hypoxia-induced cognitive and behavioral dysfunctions. Given that the hippocampus is more susceptible to hypoxic stress than other regions of the brain, this study was focused on changes in imaging parameters of this region.

\section{Materials and Methods}

\section{Ethical Approval and Animals}

58 healthy male Sprague-Dawley rats weighing $100 \mathrm{~g}$ ( 4 weeks old) were purchased from Chengdu Dashuo Biological Technology Co., Ltd., Chengdu, China, and used in the present study. This study followed the protocols of and was approved by the Experimental Animal Ethics Committee of the West China Hospital, Sichuan University, Chengdu, China (Approval No. IACUC\#20211239A). All experiments were executed in compliance with the relevant guidelines and regulations.

\section{Experimental Procedure}

The animals were randomized into two groups, including a plain group (P group rats) reared at an altitude of $500 \mathrm{~m}$ above sea level (a.s.l.) for 8 months and a plateau hypoxia group ( $\mathrm{H}$ group rats) reared on the Qinghai-Tibet Plateau, Yushu, China, at an altitude of $4250 \mathrm{~m}$ a.s.l. for 8 months ( $n=29$ for each group). All the animals were fed standard laboratory feed and water readily available in all cages ( 6 rats in each cage) at an environmental temperature of $22-24{ }^{\circ} \mathrm{C}$ and with a humidity of $50-60 \%$. The whole experimental flowchart is shown in Fig. 1.

\section{Analysis of Blood Cell Content}

After 8 months, blood samples were collected from the tail vein under isoflurane anesthesia $(n=12 /$ per group). For routine blood analysis, coccygeal vein blood $(1 \mathrm{ml})$ was 


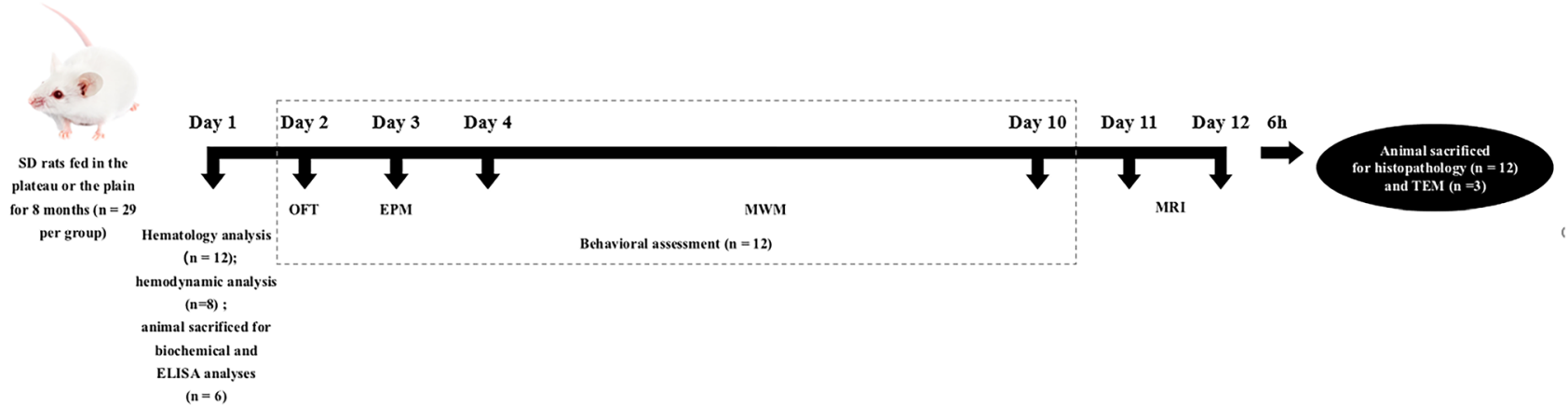

Fig. 1 Experimental flowchart. The open field test (OFT) was conducted on the 2nd day, and the elevated plus-maze (EPM) test was conducted on the 3rd day to detect potential emotional changes (e.g.,

collected with a Mindray automatic hematology analyzer (BC-2800vet, Shenzhen, China).

\section{Hemodynamic Analysis}

The rats ( $n=8 /$ per group) were subjected to measurement of mean pulmonary arterial pressure and mean systemic arterial pressure under anesthesia produced by an intraperitoneal injection of urethane $(1.0 \mathrm{~g} / \mathrm{kg}$ body weight $)$ based on the rat weight as previously described [25]. The pressure transducer was connected to the Biopac MP150, a data acquisition system, using a polystyrene microcatheter thoroughly soaked in heparin brine. After anesthesia, the right external jugular vein was shunted carefully, and the catheter was slowly pushed to the lower part. The catheter was positioned in accordance with the waveform until the typical pulmonary pressure waveform appeared. After stabilizing for $5 \mathrm{~min}$, the mean pulmonary pressure was recorded.

\section{Analysis of Oxidative Stress Parameters}

The rats in both groups ( $n=6 /$ group) were deeply anesthetized with isoflurane and then decapitated. Following the manufacturer's manual, we assessed the level of superoxide dismutase (SOD) (Cat. No. KTB1030), malondialdehyde (MDA) (KTB1050), glutathione peroxidase (GSH-Px) (KTB0640), glutathione (GSH) (KTB1600) and catalase (CAT) (KTB1040) in the hippocampus and the cortex with commercial assay kits purchased from Abbkine Scientific Co., Ltd., (CA, USA). The absorbance of SOD was determined at $450 \mathrm{~nm}$, MDA at $532 \mathrm{~nm}$, GSH-Px at $340 \mathrm{~nm}$, GSH at $412 \mathrm{~nm}$, and CAT at $540 \mathrm{~nm}$ using a Biotek $\mu$ Quant spectrofluorophotometer (Bio-Tek, Winooski, VT, USA). anxiety-like behaviors) in the $\mathrm{H}$ and $\mathrm{P}$ group rats. Performance of spatial learning and memory was measured with the Morris water maze (MWM) task on the 4th-10th days

\section{Analysis of Proinflammatory Markers}

For serum enzyme-linked immunosorbent assay (ELISA), blood samples $(3 \mathrm{ml})$ were collected from the abdominal aortic blood vessel and centrifuged $(3000 \mathrm{r} / \mathrm{min})$ for $10 \mathrm{~min}$ at $4{ }^{\circ} \mathrm{C}$ to separate the plasma from them. The supernatants thus obtained were frozen at $-80^{\circ} \mathrm{C}$ for subsequent analysis, with the highly hemolytic samples discarded. For ELISA, the whole hippocampus and part of the cortex tissues were immediately collected into labeled tubes and stored at $-80{ }^{\circ} \mathrm{C}$ until analysis. Inflammatory markers in the serum, hippocampus and cortex were assayed by ELISA using commercial kits (rat interleukin-1beta (Cat. No. ERC007), interleukin-6 (ERC003) and tumor necrosis factor-alpha (ERC102a) ELISA Kit, Neobioscience Co., Ltd., Shenzhen, China). ELISA was performed with a Biotek Epoch spectrofluorophotometer (Bio-Tek, Winooski, VT, USA) following the manufacturer's manual.

\section{Behavioral Assessment}

Behavioral tests of the animals ( $n=12$ /group) were performed from the second day after $\mathrm{H}$ group rats were transported back to Chengdu (500 $\mathrm{m}$ a.s.1.), China. The behavior test was conducted in a soundproofed and isolated room with weak illumination. The indoor temperature and ventilation were strictly controlled under the same conditions. All tests were performed between 9 am and $1 \mathrm{pm}$. The experimenter was blinded to the group division of the rats and the behavioral data analysis.

\section{Open Field Test (OFT)}

The OFT was conducted in a white plexiglass square arena $(120 \mathrm{~cm} \times 120 \mathrm{~cm} \times 40 \mathrm{~cm})$ with a black floor. The rats were placed at a stationary starting point in the center of the arena and allowed to freely explore the field for $15 \mathrm{~min}$. 
The moving traces of the animals were videotaped using an overhead camera and analyzed by a computerized analysis system. The central field, by definition, is the central area of an open space of $40 \times 40 \mathrm{~cm}^{2}$, which accounts for one quarter of the total area. After each test, the surface and sidewalls of the apparatus were thoroughly washed with $75 \%$ ethanol solution to eliminate any olfactory cues that might affect subsequent training results. The parameters measured included the duration of the travels in the central area (percentage of the total time) to evaluate anxiety-like behaviors and the total distance of the travels as a test of locomotive activity of the rats.

\section{Elevated Plus Maze (EPM) Test}

The apparatus of the EPM test was made of a black plexiglass, consisting of two opposing open arms $(50 \mathrm{~cm} \times 10 \mathrm{~cm})$ and two closed arms $(50 \mathrm{~cm} \times 10 \mathrm{~cm})$ that extended from a shared central platform $(10 \mathrm{~cm} \times 10 \mathrm{~cm})$ at $90^{\circ}$ shared by the arms. EPM was elevated by $50 \mathrm{~cm}$ above the floor. The rats were placed on the central platform with their head facing one open arm and were allowed to explore freely on the platform for $5 \mathrm{~min}$. A video camera mounted on top of the maze was used to videotape the rats. Their movements were analyzed using a computerized analysis system. The number of open arm entries and the time spent in the open arms (\% of total) were recorded to evaluate the general level of anxiety. During the intervals between the tests, the apparatus was carefully cleaned with $75 \%$ ethanol solution to remove prior odors.

\section{Morris Water Maze (MWM) Test}

The MWM is a task to evaluate the spatial learning and reference memory functions of rats, according to the procedures of Charles V. Vorhees [26]. It was conducted in a black circular pool (200-cm diameter $\times 60$-cm height) with blackinnocuous ink water (depth: $40 \mathrm{~cm}$, temperature: $25 \pm 1{ }^{\circ} \mathrm{C}$ ) midway. A $10-\mathrm{cm}$ diameter-colored target platform was placed in the fourth quadrant. The swimming tracks of the rats were recorded via a digital camera above the pool. The detailed protocol for cued learning, training and probe trials is described as follows.

(1) Cued learning test. On the first day, the swimming ability and visual state of rats in the two groups were detected. The platform was raised approximately $1.5-2 \mathrm{~cm}$ above the water surface. The rats entered the water from any random quadrant to find the platform and then rested on the platform for $30 \mathrm{~s}$. (2) Training test. The rats were randomly put into the pool facing the wall at four water entry points, including the northeast, northwest, southeast, and southwest. In each experiment, each rat spent $60 \mathrm{~s}$ at most finding the hidden platform $1.5-2 \mathrm{~cm}$ beneath the water surface. Once the rats found the platform, they were allowed to sit on it for $10 \mathrm{~s}$. The time the rats took from entering the water to finding the platform and climbing onto the platform was recorded as the escape latency. If the rats failed to climb onto the platform within the given time, the time was recorded as $60 \mathrm{~s}$. After that, the rats were guided to stay on the platform for $15 \mathrm{~s}$. Following this protocol, each animal was trained four times per day for 5 consecutive days. (3) Probe test. The platform was removed after the training test (on the 7 th day), and then the animals were put into the water from the opposite quadrant to trace their movement trajectory for $60 \mathrm{~s}$. The time, distance, speed of swimming and number of crossings of the original platform position in the target quadrant (the quadrant where the platform was originally placed) were recorded.

\section{MRI Acquisition}

MRI scanning was conducted after the behavior assessment ( $n=12$ /group). The small animal MRI examination was performed in a 7.0 T magnetic resonance imaging system (BioSpec 70/30; Bruker, Karlsruhe, Germany). For the animal study, a 72-mm open birdcage quadrature volume resonator was utilized for excitation, and a four-channel $(2 \times 2)$ phased array coil was used as a receiver. Anesthesia was initially induced with $2.5 \%$ isoflurane and was maintained with $1 \%$ to $1.5 \%$ isoflurane ( $2 \mathrm{~L} / \mathrm{min}$ oxygen flow) during MRI scanning. The rats were placed in a prone position with their head fixed using a tooth rod and two ear pins. A respiratory rhythm sensor was installed beneath the abdomen to observe and monitor respiration at $50-80$ breaths per minute. Additionally, the rats were kept on an animal heating pad connected to hot circulating water at $37 \pm 1.0^{\circ} \mathrm{C}$ during scanning to prevent the body temperature from falling. Multiparametric MR image scanning (including high-resolution T2, CEST, T1 map and DCE) was sequentially performed on each rat.

For VBM, high-resolution anatomical MRI data were captured using a T2-weighed turbo-rapid acquisition with relaxation enhancement (RARE) sequence. T2-weighted images (T2WI) were acquired using a two-dimensional RARE sequence with parameters including repetition time $(\mathrm{TR})=11,400 \mathrm{~ms}$, echo time $(\mathrm{TE})=48 \mathrm{~ms}$, number of averages $=6$, slice thickness $=0.35 \mathrm{~mm}$, slice gap $=0 \mathrm{~mm}$, field of view $(\mathrm{FOV})=35 \times 35 \mathrm{~mm}^{2}$, in-plane resolution of 0.137 $\mathrm{mm}^{2} \times 0.137 \mathrm{~mm}^{2}$ (matrix size $=256 \times 256$ ), and total scanning time for T2WI of approximately $38 \mathrm{~min}$.

When the high-resolution T2-weighted images of each rat scanning were obtained, other sequence scans were performed. For CEST, the $\mathrm{B}_{0}$ field over the whole rat brain was corrected using field mapping following higher-order shimming procedures. Continuous-wave CEST data were acquired using a fat-suppressed RARE sequence with an 
RF saturation pulse ( $T R=5 \mathrm{~s}, \mathrm{TE}=30.73 \mathrm{~ms}$, RARE factor $=24$, matrix size $=96 \times 96$, slice thickness $=1.5 \mathrm{~mm}$, $\mathrm{FOV}=30 \times 30 \mathrm{~mm}^{2}$ ). The RF saturation power (B1) and saturation length for CrCEST were $2.0 \mu \mathrm{T}$ and $1 \mathrm{~s}$, respectively [27]. The frequency measured to assess CrCEST ranged from 1 to $3.5 \mathrm{ppm}$ at an interval of $0.05 \mathrm{ppm}$. The reference image $\left(\mathrm{S}_{0}\right.$ image) was obtained by setting the offset at $200 \mathrm{ppm}$ [28]. Quantitative T1 relaxation maps were acquired using RARE with variable repetition time sequences with seven TRs $(0.5,1,1.5,2,3.5,5$, and $8 \mathrm{~s})$, $\mathrm{TE}=12 \mathrm{~ms}$, RARE factor $=4$. All CrCEST scans were selected from a single slice that was parallel to the coil and set to approximately $-2.36 \mathrm{~mm}$ (according to the results of VBM) in relation to the anterior commissure (AC).

For DCE-MRI, 120 continuous dynamic T1_FLASH images were acquired. The animals were manually intravenously given a bolus of gadopentetate dimeglumine as a contrast agent $(0.2 \mathrm{mmol} / \mathrm{kg}$, BeiLu Pharmaceutical Co., Ltd., Beijing, China) via the caudal vein after 10 to 15 baseline images (to cover 10 to $15 \mathrm{~s}$ of acquisition), and precontrast images were acquired. All DCE-MRI scans were selected as double slices that were parallel to the coil positioned and set to approximately $-2.36 \mathrm{~mm}$ and $-4.70 \mathrm{~mm}$ in relation to the AC. The scanning parameters included $\mathrm{TR}=15.6 \mathrm{~ms}$, $\mathrm{TE}=1.8 \mathrm{~ms}, \mathrm{FOV}=35 \times 35 \mathrm{~mm}^{2}$, flip angle of $12^{\circ}$, slice thickness of $0.8 \mathrm{~mm}$ and in-plane resolution of $0.273 \times 0.273$ $\mathrm{mm}^{2}$.

\section{MRI Data Processing}

VBM: High-resolution T2-weighted imaging was performed using the statistical parametric mapping toolbox and software (SPM12, Wellcome Trust Centre for Neuroimaging, London, UK) in MATLAB 2013b. (1) Bet: High precise whole-brain masks for segmenting the nonbrain tissue for in vivo scans were achieved using PCNN3D [29], and the whole brain tissues were extracted with an image calculator in the SPM12 toolbox with the calculated approach to the rat's unprocessed scans multiplied by their brain mask. An improper mask was embellished using ITK-SNAP [30]. (2) Origin setting and voxel size resizing: Each skull-stripped T2-weighted image was resized by a factor of 10 to account for variation in the whole-brain volume between humans and rodents [31]. Meanwhile, the resized T2-weighted images were manually reoriented to the origin. (3) Segmentation and spatial normalization: In the old segment batch in SPM12, the resized and reoriented T2-weighted images were segmented into tissue probability maps of the gray matter, white matter and cerebrospinal fluid with a unified segmentation approach [12]. Then, the tissue class images were nonlinearly normalized to the SIGMA template [32], and the signal intensities of the normalized maps were modulated by the Jacobian determinant to add the transformation information of regional or global volume generated by nonlinear transformations (such as warp and deform) in image segmentation results to account for voxelwise volume alteration in the cerebral regions [33] (Supplemental Fig. S1). (4) Smooth: The images were then smoothed with an 8-mm full-widthat-half-maximum Gaussian kernel for analysis. (5) Visualization of statistical analysis and results: After the obtained images were imported into the statistical analyzer in SPM12, contrast T-maps were generated and compared voxel by voxel with two-sample $t$ tests with a gray matter mask settled at an absolute threshold mask of 0.2 (with relative total brain volumes entered as a covariate). The more stringent familywise error correction was used for multiple comparisons, with a statistical threshold of 0.05 and with a cluster size of 200 voxels. However, although image preprocessing was performed on the resized scales, the results of VBM analysis were still displayed on the original scales. The significantly altered clusters in the hippocampus were saved as masks, and their corresponding rGMV was extracted using the dpabi toolbox (http://rfmri.org/dpabi).

Rat CEST data processing was performed using customwritten MATLAB scripts (MathWorks, www.mathworks. com, version R2018b). Regions of interest (ROIs) were manually delineated on the left and right hippocampi of $\mathrm{P}$ and $\mathrm{H}$ group rats. The CrCEST peaks $(R)$ in the $R$-spectrum were extracted using the polynomial and Lorentzian lineshape fitting (PLOF) method as described previously [28, $34,35]$. Cr concentrations were quantified by the PLOF scheme based on the principle that the values of CrCEST peaks extracted from the $\mathrm{Z}$ spectrum are proportionate to the $\mathrm{Cr}$ concentration. CrCEST peaks can be represented by a Lorentzian function as follows:

$R=R_{\text {exch }} \frac{(w / 2)^{2}}{(w / 2)^{2}+\left(\Delta-\Delta_{\text {exch }}\right)^{2}}$

where $\mathrm{w}$ is the peak line width (full-width-at-half-maximum) of the Lorentzian line shape, $\Delta$ is the offset, $\Delta_{\text {exch }}$ is the chemical shift of the CrCEST peak (approximately $1.95 \mathrm{ppm}$ in vivo) in relation to the water signal, and $R_{\text {exch }}$ is the true apparent relaxation rate of the CEST peaks in the $R$ - spectrum.

The broad background $\left(R_{\text {back }}\right)$ in the $R$-spectrum can be represented by a mixed polynomial and Lorentzian function as follows:

$R_{\mathrm{back}}=\frac{\mathrm{C} 0(\mathrm{C} 1 / 2)^{2}}{(\mathrm{C} 1 / 2)^{2}+\Delta \omega^{2}}+C 2+C 3 \cdot \Delta \omega$

A broad background signal $\left(R_{\text {back }}\right)$, including direct water saturation (DS), magnetization transfer contrast (MTC), aromatic protons and other metabolites, was fitted by a higherorder polynomial function. The first Lorentzian function in Eq. (2) was used to account for DS, whereas $\mathrm{C} 2+\mathrm{C} 3 \bullet \Delta \omega$ 
was used to fit MTC and other exchanging protons, such as amines and hydroxyls.

DCE: The Ktrans value was calculated using DCE@ urLAB 1.0 software for Microsoft Windows 7 (Microsoft, Redmond, WA, USA; http:/www.die.upm.es/im/archives/ DCEurLAB/). All DCE images were analyzed as described previously [36]. To evaluate BBB permeability with DCE contrasts, two experienced observers blinded to each animal's status manually delineated the borders of the ROIs in the hippocampus and the ipsilateral cortex of both hemispheres in slices 1 (AC, $-2.36 \mathrm{~mm}$ ) and 2 (AC, $-4.70 \mathrm{~mm}$ ), as shown in Supplemental Fig. S2, and prominently visible blood vessels were excluded based upon the T2-weighted images. Finally, the extended Tofts-Kety model including the parameter of the plasma volume was chosen to obtain the Ktrans values averaged for each rat. For each rat, the Ktrans value of the hippocampus or the cortex was calculated as a whole as the average of the Ktrans values of four ROIs of the two slices.

\section{Histopathological Examination}

Following MRI, the brains of all the animals were collected and underwent histological study. The animals were euthanized with an overdose of isoflurane and perfused transcardially with $250 \mathrm{ml}$ of ice-cold saline, followed by $300 \mathrm{ml}$ of $4 \%$ paraformaldehyde (PFA) in phosphate-buffered saline (PBS, $0.1 \mathrm{~mol} / \mathrm{L}, \mathrm{pH} \mathrm{7.4)}$ for $30 \mathrm{~min}$ at a rate of $12 \mathrm{~mL} /$ min. After perfusion, the brains were removed and fixed in $40 \mathrm{ml}$ of $4 \%$ paraformaldehyde. After sufficient postfixation, the brains were embedded in paraffin. Four-micrometer-thick coronal sections were consecutively taken from approximately bregma $-2.36 \mathrm{~mm}$ by sledge microtome, which included the focus of the decreased rGMV values in the hippocampus observed in the $\mathrm{H}$ group compared with that in the $\mathrm{P}$ group.

Nissl Staining The obtained tissue sections (six brains/ group) were collected on glass slides, deparaffinized, stained with $1 \%$ cresyl violet solution by Nissl staining for morphological study of the hippocampus, and then examined under a light electric microscope (CM 3000; Leica, Bensheim, Germany).

Fluoro Jade-B Staining For the neurodegeneration study, brain sections of the rats were stained with Fluoro Jade$\mathrm{B}$ (FJB) dye, which is a fluorescent substance that binds sensitively and specifically to degenerating neurons [37]. Staining was performed according to the method of Schmued et al. [38]. After staining, degenerating neurons were counted under a fluorescence microscope (Nikon Eclipse C1, Tokyo, Japan). Images of CA1, CA3 and ventral dentate gyrus (DG) granule cells (upper lobe) were obtained at $40 \times$ magnification. The upper blade of DG granule cells showed neuronal susceptibility to hypoxia compared with the dorsal blade (lower blade) [39]. Two independent observers blinded to the group division of the rats used 'Image-Pro Plus 6.0' image analysis software (MediaCybernetics, Silver Spring, MD, USA.), manually counted the positive neurons of FJB in the hippocampal subregions of interest (CA1, CA3, and DG) per rat section with six rats per group $(n=6$ rats/group) and each area in 3 to 5 sections.

Immunofluorescence Staining Levels of antiglial fibrillary acidic protein (GFAP, 1:500, Wuhan Servicebio Biotechnology Co., Ltd., Wuhan, China), anti-ionized calcium binding adaptor molecule 1 (Iba-1, 1:1000, Ibid.) and antineuronal nuclei (1:1000, Ibid.) were used to detect astrocytes, microglia and neurons. After the paraffinized brain slides were dewaxed and rehydrated, microwave heat-induced antigens were retrieved with citrate $(\mathrm{pH} 9.0)$ for $15 \mathrm{~min}$ before they were incubated in $1 \mathrm{~N}$ hydrochloric acid $(1 \mathrm{~mol} / \mathrm{L})$ for $20 \mathrm{~min}$ and fixed in 4\% PFA for $10 \mathrm{~min}$. Next, the sections were blocked with $3 \%$ BSA for $30 \mathrm{~min}$ at room temperature and incubated with a primary antibody in blocking serum at $4{ }^{\circ} \mathrm{C}$ overnight. The following day, the sections were incubated with secondary antibodies, including Alexa Fluor 488 goat anti-mouse and $\mathrm{Cy} 3$ goat anti-rabbit antibodies, for $1 \mathrm{~h}$ in the dark at room temperature. The cell nucleus was counterstained with 4,6-diamidino-2-phenylindole (DAPI) for $10 \mathrm{~min}$ in the dark. Each of the above sections was washed three times for $5 \mathrm{~min}$ with PBS. The slides were observed under an immunofluorescence microscope (Nikon Eclipse C1, Tokyo, Japan). GFAP-labeled and Iba1labeled cells were counted in the hippocampal subregions of interest (CA1, CA3, and DG) per rat section for six rats per group ( $n=6$ rats/group), and each area was counted in $3-5$ sections. The counting was performed by 2 independent observers blinded to the group division of the rats.

Golgi-Cox Staining The modified Golgi-Cox staining method was used to detect the dendritic spines of hippocampal neurons as described previously [40]. Intracardial perfusion was performed with $500 \mathrm{ml}$ of $0.9 \%$ saline at room temperature in rats deeply anesthetized with isoflurane. Immediately after perfusion, the brains were fixed in bottles of $50 \mathrm{ml}$ filled with $40 \mathrm{ml}$ of $4 \%$ PFA for $24 \mathrm{~h}$. Then, the brains were impregnated, sectioned coronally $(100 \mu \mathrm{m})$ containing the hippocampus and the cortex, and stained according to the instructions in the FD Rapid Golgi Stain Kit. Dendrite spine density and dendrite complexity were assayed using ImageJ software as described previously [41]. Dendrite spine densities under a $100 \times$ oil objective lens were counted by the number of spines along 30-90 $\mu \mathrm{m}$ segments of the second- or third-order dendritic branch arbitrarily selected in apical dendrites in hippocampal and cortical 
neurons (spine density $=$ number of dendritic spines/length of dendrites $\times 10$ ). Sholl analysis under a $20 \times$ oil objective lens was performed to evaluate the dendrite complexity by counting the number of intersections between the dendrites with an overlaid concentric grid at an interval of $10 \mu \mathrm{m}$. Three neuronal cells per brain slice and three brain slices per animal ( $\mathrm{n}=6$ rats/group) were chosen for quantitative analysis. An observer blinded to the group division of the rats analyzed the number of dendritic spines with ImageJ (National Institutes of Health, Bethesda, MD, USA).

TdT-Mediated dUTP Nick-End Labeling (TUNEL) Assay Apoptosis was quantified to observe DNA fragments by TUNEL reaction. After being dewaxed and rehydrated, the paraffin-embedded slices were incubated with proteinase $\mathrm{K}$ for $30 \mathrm{~min}$. After incubation, the sections were washed in $0.1 \mathrm{M}$ PBS for $5 \mathrm{~min}$ and then incubated in a permeabilized working solution at room temperature for $20 \mathrm{~min}$. The sections were slightly dried and then incubated with a mixture of terminal deoxynucleotidyl transferase enzyme and digoxigenin-tagged dUTP for $2 \mathrm{~h}$. Subsequently, the cell nuclei were counterstained using DAPI, and the slices were finally mounted with anti-fade mounting medium. TUNEL-positive neurons were counted by two blinded experimenters in the hippocampal subregions of interest (CA1, CA3, and DG) per rat section for six rats per group ( $n=6$ rats/group) and each area in 3-5 sections $(\times 200)$.

\section{Transmission Electron Microscopy (TEM)}

The ultrastructural changes in the hippocampus (CA1, CA3, and DG) and the cortex were observed using TEM. The rats $(\mathrm{n}=3$ rats/group) were deeply anesthetized using isoflurane as mentioned above, and the brains were perfused with $2.5 \%$ glutaraldehyde perfusion solution via the heart. We used a blade to cut and harvest hippocampal and cortical tissue blocks quickly (within $1 \mathrm{~mm}^{3}$ ). The removed tissue was immediately fixed in 3\% glutaraldehyde (buffered at $\mathrm{pH}$ 7.4) for $18-20 \mathrm{~h}$ and refixed in $1 \%$ osmium tetroxide for $2 \mathrm{~h}$ at $48{ }^{\circ} \mathrm{C}$. Following dehydration, the hippocampus and the cortex were embedded in acetone and epoxy resin (Epon 812), and ultrathin sections of $50 \mathrm{~nm}$ were fixed on cuprum grids. After staining with $1 \%$ uranyl acetate and lead citrate at room temperature, the ultrastructure of hippocampal and cortical neurons was observed using a JEM-1400FLASH (JEOL Ltd., Tokyo, Japan) TEM.

\section{Statistical Analysis}

Statistical analysis was performed using Prism Version 8.0 software (GraphPad Software, La Jolla, CA, USA). All data are expressed as histograms of means \pm SD values. The two groups were compared using unpaired two-tailed Student's $t$ test or a Mann-Whitney nonparametric test according to data normality test confirmed by quantile-quantile plot. For the MWM test and Sholl analysis, two-way repeated ANOVA followed by Sidak's post hoc test was used. Pearson's correlation coefficient $(r)$ between behavioral parameters and MRI parameters was calculated. A value of $P<0.05$ was considered to be statistically significant.

\section{Results}

\section{Body Weight, Blood Routine and Arterial Pressure}

After 8 months of exposure to plateau hypoxia, the body weight of the $\mathrm{H}$ group rats was higher than that of the $\mathrm{P}$ group rats $(P<0.05)$. Red blood cells, hemoglobin, white blood cells and granulocyte counts were significantly higher in the $\mathrm{H}$ group than in the $\mathrm{P}$ group $(P<0.05)$. The mean pulmonary arterial pressure of the $\mathrm{H}$ group was significantly higher than that of the $\mathrm{P}$ group $(P<0.05)$, whereas the mean systemic arterial pressure of the $\mathrm{H}$ group was not influenced by exposure to high-altitude hypoxia $(P>0.05)$ (Table 1$)$, as we expected.

Table 1 Body weight, full blood count, and hemodynamic parameters of both groups

\begin{tabular}{lccr}
\hline & $\mathrm{H}(\mathrm{n}=12)$ & $\mathrm{P}(\mathrm{n}=12)$ & $P$ value \\
\hline Weight & & & \\
Before plateau hypoxia $(\mathrm{g})$ & $99 \pm 5$ & $100 \pm 3$ & 0.134 \\
After plateau hypoxia $(\mathrm{g})$ & $665 \pm 63$ & $620 \pm 36$ & 0.032 \\
Hematological analysis & & & \\
WBC (million/mm $\left.{ }^{3}\right)$ & $8.6 \pm 2.1$ & $4.7 \pm 1.7$ & 0.001 \\
Lymphocyte (\%) & $41.9 \pm 10.4$ & $57.1 \pm 19.2$ & 0.061 \\
Monocyte (\%) & $5.5 \pm 3.2$ & $4.9 \pm 2.5$ & 0.681 \\
Granulocyte (\%) & $51.7 \pm 9.1$ & $36.5 \pm 17.4$ & 0.041 \\
RBC (million/mm $\left.{ }^{3}\right)$ & $11.6 \pm 0.5$ & $10.3 \pm 0.4$ & $<0.001$ \\
MCV (\%) & $46.2 \pm 9.5$ & $46.9 \pm 1.3$ & 0.824 \\
Hb (g/dl) & $164.3 \pm 9.2$ & $135.4 \pm 4.4$ & $<0.001$ \\
Hemodynamic parameters & & & \\
PAPm (mmHg)* & $22.9 \pm 5.2$ & $15.8 \pm 1.6$ & 0.0013 \\
SAPm (mmHg)* & $97.2 \pm 13.6$ & $100.8 \pm 18.4$ & 0.6818 \\
\hline
\end{tabular}

Values are expressed as the mean $\pm \mathrm{SD}$. Lymphocytes, monocytes and granulocytes expressed in \%

$W B C$ white blood cells (million $/ \mathrm{mm}^{3}$ ), $R B C$ red blood cells (million/ $\left.\mathrm{mm}^{3}\right), M C V$ mean cell volume (\% volume), $H b$ hemoglobin $(\mathrm{g} / \mathrm{dl})$, $P A P m$ mean pulmonary arterial pressure, $S A P m$ mean systemic arterial pressure, $H$ plateau hypoxia, $P$ plain

$* \mathrm{n}=8$ per group 


\section{Oxidative Stress Parameters in the Hippocampus and the Cortex}

The levels of MDA in the hippocampal and cortical tissues of the $\mathrm{H}$ group were significantly higher than those of the $\mathrm{P}$ group $(P<0.05)$. The activities of GSH and antioxidant enzymes (SOD, CAT, GSH-Px) in the hippocampal and cortical tissues of $\mathrm{H}$ group rats decreased significantly compared with that of $\mathrm{P}$ group rats $(P<0.05)$ (Table 2$)$.

\section{Proinflammatory Cytokine Levels in the Blood, Hippocampus, and Cortex}

ELISA results in Fig. 2a-i show that, compared with that of the $\mathrm{P}$ group, the levels of tumor necrosis factor-alpha, interleukin-6, interleukin-1beta in the blood, hippocampus and cortex of the $\mathrm{H}$ group increased significantly $(P<0.05)$.

\section{Behavioral Test Results}

\section{Plateau Hypoxia Exposure Induced Anxiety-Related Behaviors}

As shown in Fig. 3, compared with the $\mathrm{P}$ group rats, the $\mathrm{H}$ group rats spent less time in the central square of the OFT, whereas the total distance traveled by the $\mathrm{H}$ group rats remained unaltered (Fig. 3a and b). EPM results showed that $\mathrm{H}$ group rats spent less time in and had fewer entries into the open arms, whereas the total number of arm entries remained unaltered (Fig. 3c-e). All these data suggest that plateau hypoxia exposure could induce anxiety-like behaviors in rats.

\section{Plateau Hypoxia Exposure Induced Reduced Spatial Memory}

In the visible platform trial, no significant differences in escape latency (H group rats: $45.22 \pm 8.40$ vs. $\mathrm{P}$ group rats: $42.93 \pm 11.80, P>0.05$ ) or swimming speed (H group rats: $12.16 \pm 6.59$ vs. $P$ group rats: $16.29 \pm 6.82, P>0.05)$ were found between the two groups (Fig. 3f and g). Repeated measurements with ANOVA for the escape latency and traveled distance to find the hidden platform across the training days indicate a significant effect on the groups $[F(1,22)=29.35 ; P<0.0001$ for the escape latency and $F(1,22)=27.10 ; P<0.0001$ for the traveled distance $]$ and training days $[F(3.142,69.12)=28.32 ; P<0.0001$ for the escape latency and $F(3.075,67.64)=31.54 ; P<0.0001$ for the traveled distance], with a significant interactive effect of the two $[F(4,88)=2.56 ; P=0.044$ for the escape latency and $F(4,88)=2.63 ; P=0.039$ for the traveled distance $]$. Sidak's multiple comparisons revealed that the escape latency and distance traveled to reach the platform significantly increased in the $\mathrm{H}$ group rats compared with the $\mathrm{P}$ group rats on the 4 th and 5 th days of the learning trials (Fig. 3 h and i). Probe trials were conducted to assess spatial memory performance at $24 \mathrm{~h}$ after the 5 th day of training. $\mathrm{H}$ group rats spent less time percentage than $\mathrm{P}$ group rats in the target quadrant of the pool, in which the hidden platform had been located beforehand ( $\mathrm{H}$ group rats: $23.11 \pm 2.54 \%$ vs. $\mathrm{P}$ group rats: $35.04 \pm 1.59 \%, P<0.01$ ) (Fig. $3 \mathrm{j}$ ) and $\mathrm{H}$ group rats made fewer platform crossings than $\mathrm{P}$ group rats did (H group rats: $1.92 \pm 1.44$ vs. $P$ group rats: $3.17 \pm 1.34, P<0.05)$ (Fig. 3k). All these data suggest that plateau hypoxia exposure decreased the spatial memory performance of $\mathrm{H}$ group rats in comparison with that of $\mathrm{P}$ group rats.

\section{Multimodal MR Imaging Findings}

\section{Whole-Brain VBM}

As expected, $\mathrm{H}$ group rats exhibited decreased rGMV $(P<0.05$, familywise error-corrected) in their hippocampus compared with $\mathrm{P}$ group rats (Table 3; Fig. 4), which was also found in the primary somatosensory cortex of $\mathrm{H}$ group rats (Ibid.).
Table 2 Effect of chronic exposure to a plateau hypoxic environment on oxidative stress parameters in the hippocampus and the cortex of $\mathrm{H}$ and $\mathrm{P}$ group rats

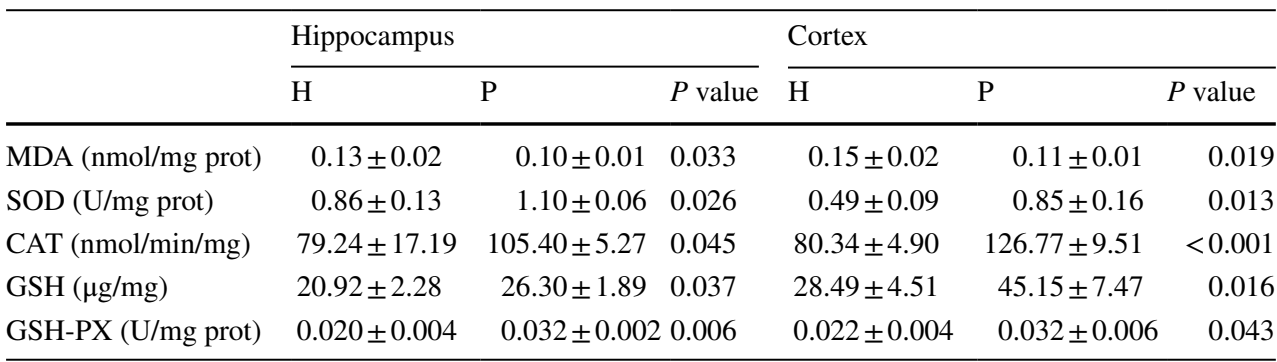

Values are expressed as the mean $\pm \operatorname{SD}(n=6$ per group)

$M D A$ malondialdehyde, GSH glutathione, $S O D$ superoxide dismutase, CAT catalase, GSH-Px glutathione peroxidase, $H$ plateau hypoxia, $P$ plain 

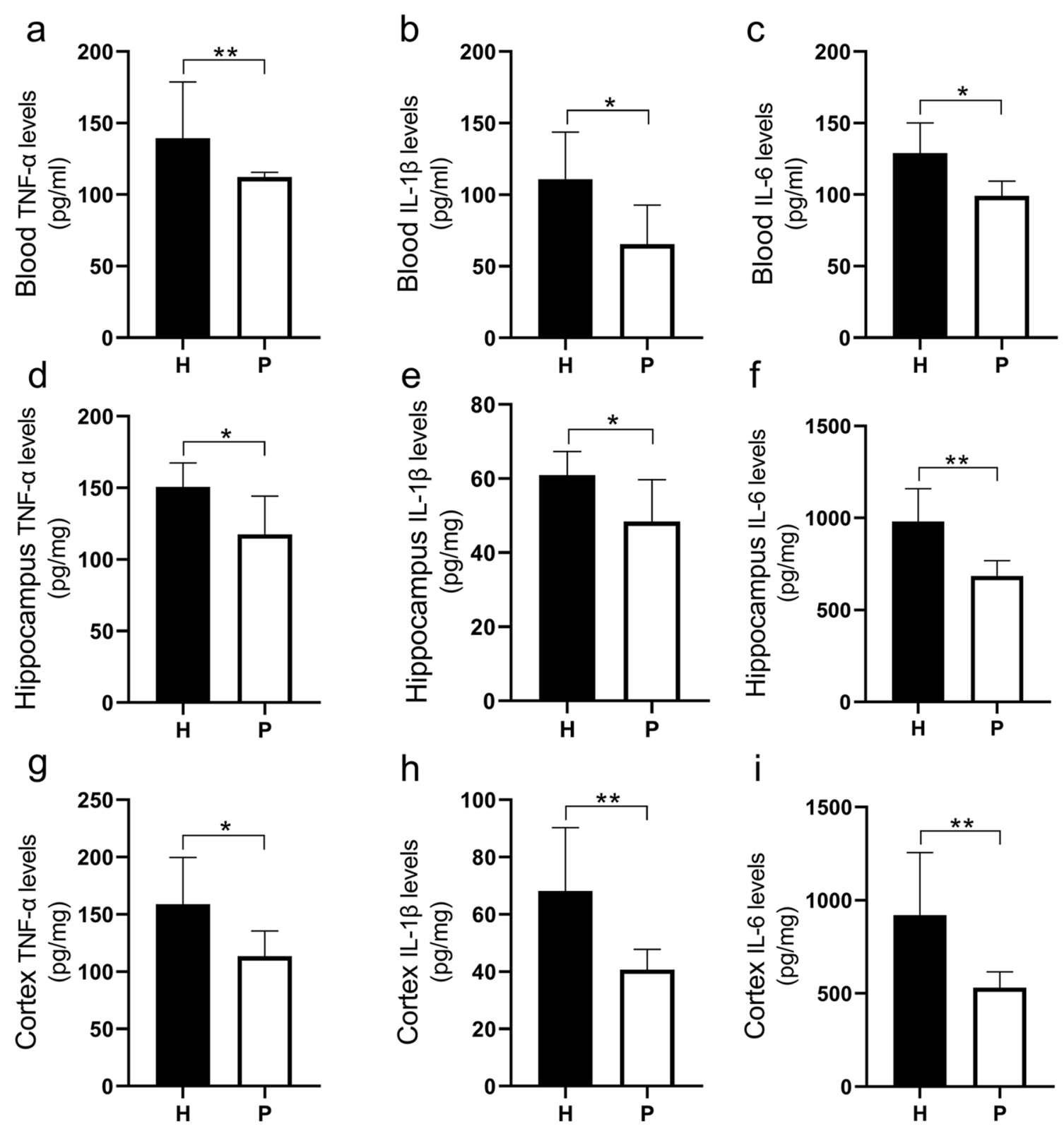

Fig. 2 Bar graphs show between-group differences in TNF- $\alpha$ (a, d, and $\mathbf{g}$ ), IL-1 $\beta$ (b, e, and $\mathbf{h}$ ), and IL-6 (c, f, and i) in the blood, hippocampus and cortex tissue. Data are expressed as the mean \pm SD

(n=6 per group). $H$ plateau hypoxia, $P$ plain, $T N F-\alpha$ tumor necrosis factor-alpha, $I L-1 \beta$ interleukin-1beta, $I L-6$ interleukin-6. ${ }^{*} P<0.05$, $* * P<0.01$

\section{CrCEST}

Apparent relaxation rates of $\mathrm{Cr}\left(R_{\mathrm{Cr}}\right)$ maps of the brains of the two groups of rats as well as the corresponding $\mathrm{S}_{0}$ images are shown in Fig. 5a and b. Typical CrCEST Z-spectra of the hippocampus (black ROI 1) and the cortex (black ROI 2 ) in $\mathrm{H}$ and $\mathrm{P}$ group rats are plotted in Fig. $5 \mathrm{c}$ along with the PLOF fitting curves. We selected the averaged $R_{\mathrm{Cr}}$ to represent CrCEST data. $R_{\mathrm{Cr}}$ is linearly related to the Cr concentration, indicating that $R_{\mathrm{Cr}}$ is not susceptible to the scale-down effect induced by DS and MTC in CEST contrast imaging
$[28,35] . R_{\mathrm{Cr}}$ values in the hippocampus $\left(0.062 \pm 0.018 \mathrm{~s}^{-1}\right.$ vs. $0.110 \pm 0.012 \mathrm{~s}^{-1}, P<0.001$ ) (Fig. 5d) and the cortex of $\mathrm{H}$ group rats $\left(0.049 \pm 0.033 \mathrm{~s}^{-1}\right.$ vs. $0.087 \pm 0.045 \mathrm{~s}^{-1}$, $P<0.05$ ) (Fig. 5e) decreased significantly compared with that of $\mathrm{P}$ group rats.

\section{DCE-MRI}

Quantitative analysis of Ktrans maps of $\mathrm{H}$ and $\mathrm{P}$ group rats is shown in Fig. 6a and b. Compared with $\mathrm{P}$ group rats, $\mathrm{H}$ group rats showed significantly higher Ktrans values in the 

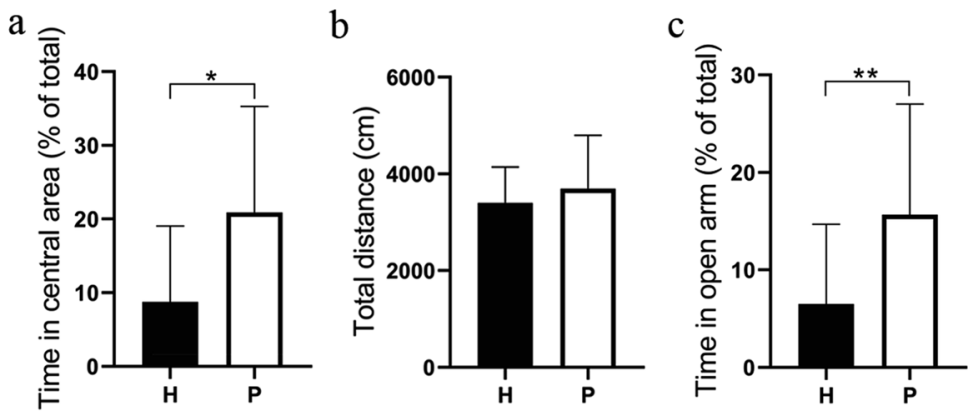

d

e

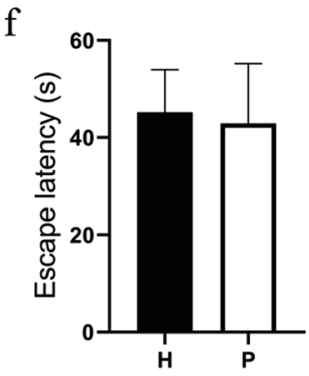

$\mathrm{g}$
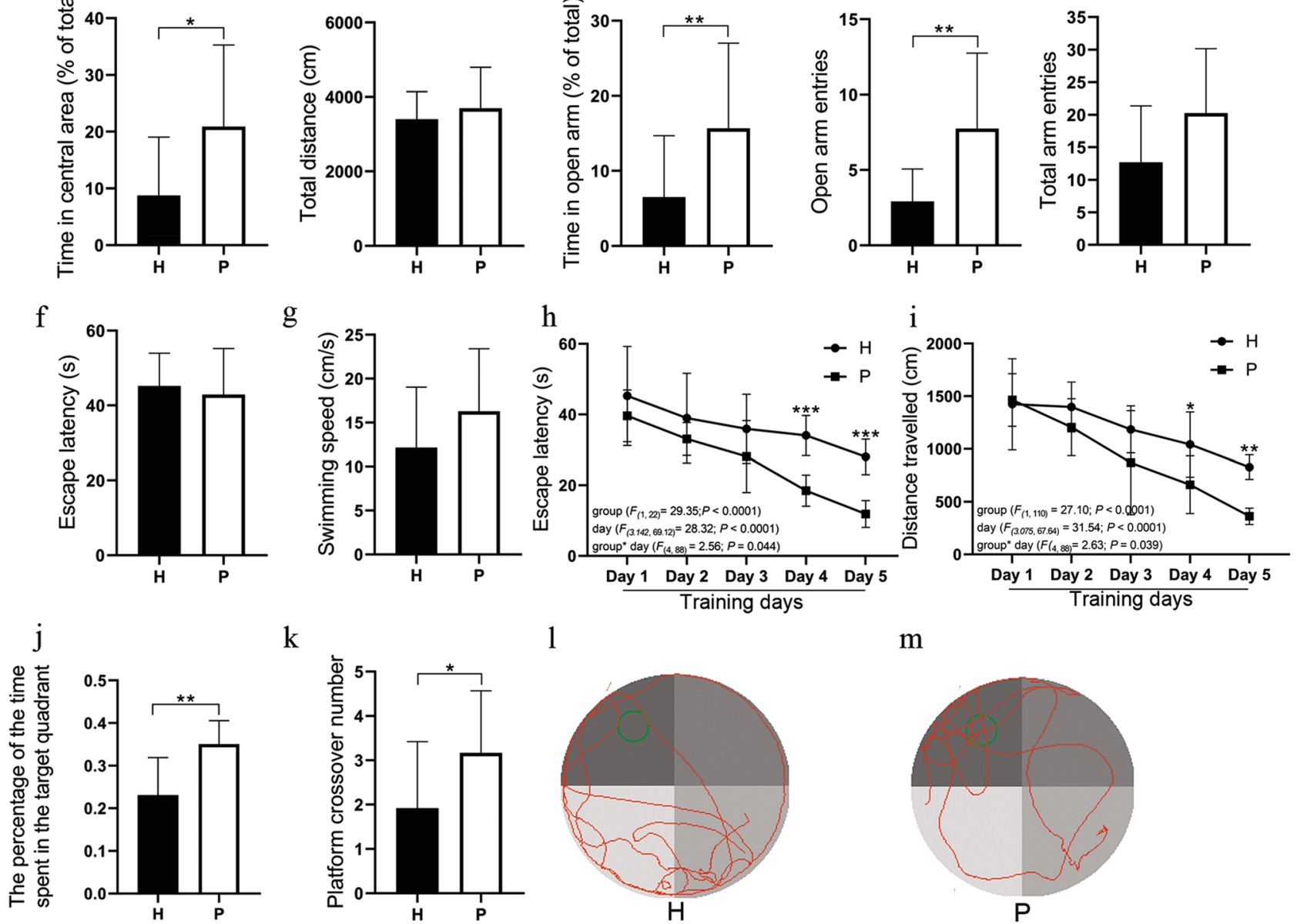

$\mathrm{k}$

1
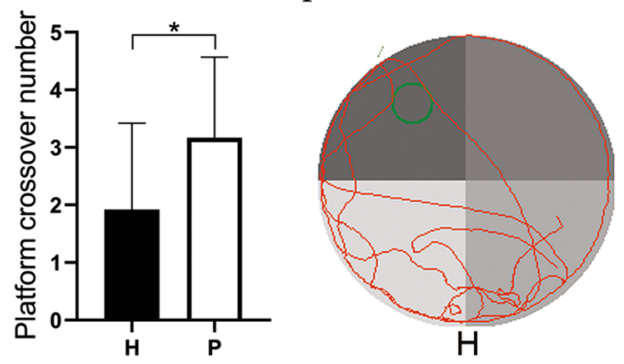

$\mathrm{m}$

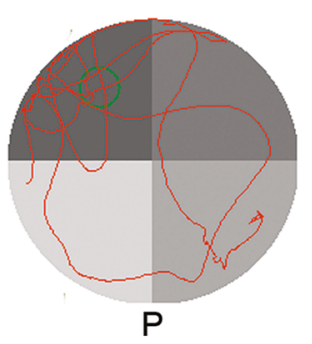

Fig. 3 Between-group differences in the results of the OFT (a, b), EPM (c-e), and MWM (f-m). In the OFT, time in the central area (\% of total) (a) and total distance traveled (b) were obtained. In the EPM test, time in open arms (\% of total) (c), open arms entries (d), and total arm entries (e) were obtained. In the visible platform trial of the MWM, escape latency (f) and swimming speed (f) to the platform over water in the target quadrant were obtained. In the hidden platform trial of the MWM, the escape latency (h) and distance traveled (i) to the underwater platform in the target quadrant were obtained

during the 2-6 day training phase. The times of crossing the original platform location (j) and time spent in the target quadrant (\% of total) (k) were obtained during the probe trial of the MWM on the 7th day. Representative swimming paths of rats during the probe trial on Day 7 are depicted $(\mathbf{l}$ and $\mathbf{m})$. Data are expressed as the mean $\pm \mathrm{SD}(\mathrm{n}=12$ per group). $H$ plateau hypoxia, $P$ plain, $O F T$ open field test, $E P M$ elevated plus maze, $M W M$ Morris water maze. $* P<0.05, * * P<0.01$, $* * * P<0.001$

Table 3 The peak of the local maxima within each significant cluster $(P<0.05$, familywise error-corrected $)$ showed significant regional gray matter volume decreases in $\mathrm{H}$ group rats compared with those in $\mathrm{P}$ group rats

\begin{tabular}{|c|c|c|c|c|}
\hline Brain regions & Coordinates $(\mathrm{x}, \mathrm{y}, \mathrm{z})$ & Peak intensity & Z score & Voxels \\
\hline Hippocampus (L) & $(-0.6,3.59,-2.36)$ & -8.6411 & 6.20 & 2710 \\
\hline $\begin{array}{l}\text { Primary somatosensory cortex fore- } \\
\text { limb (R) }\end{array}$ & $(1.5,3.59,-9.60)$ & -6.8465 & 5.36 & 2842 \\
\hline
\end{tabular}

The atlas coordinates of the peaks are relative to bregma in the medial-lateral (x), superior-inferior (y), and anterior-posterior (z) directions $(\mathrm{mm})$

$H$ plateau hypoxia, $P$ plain 
Fig. 4 Whole-brain voxel-based morphometry analysis results. Red-to-yellow colors represent decreased gray matter volume after plateau hypoxia exposure $(\mathrm{n}=12$ per group; familywise error-corrected $p<0.05$ ). Color bar units refer to t-scores

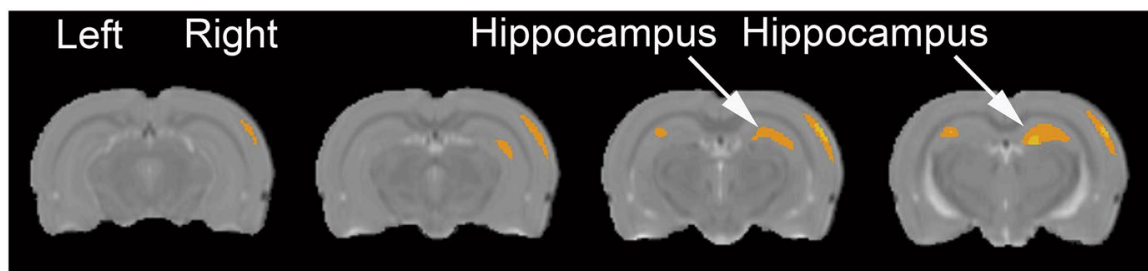

\section{t- scores}
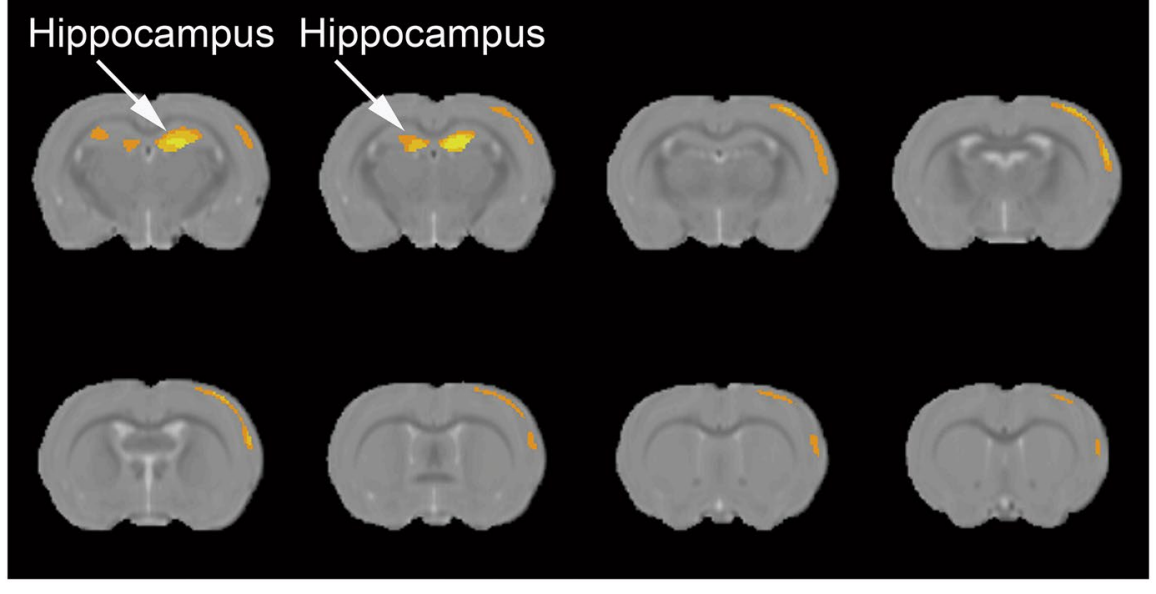

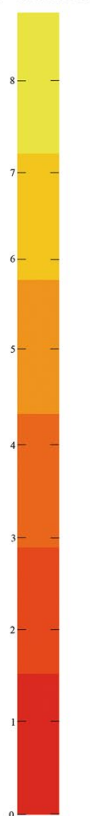

hippocampus $\left(0.221 \pm 0.060 \mathrm{~min}^{-1}\right.$ vs. $0.086 \pm 0.033 \mathrm{~min}^{-1}$, $P<0.001)$ (Fig. 6c) and the cortex $\left(0.253 \pm 0.092 \mathrm{~min}^{-1}\right.$ vs. $0.149 \pm 0.030 \mathrm{~min}^{-1}, P<0.01$ ) (Fig. 6d).

\section{Correlation Analysis}

As shown by the correlation analysis results in Fig. 7, rGMV of the hippocampus was positively correlated with the number of platform crossings ( $r=0.644, P<0.05)$, time spent in the target quadrant ( $\%$ of total) $(r=0.571, P<0.05)$, time spent in the open arm $(r=0.603, P<0.05)$ and time spent in the central area (\% of total) $(r=0.687, P<0.05)$. The $R_{\mathrm{Cr}}$ of the hippocampus was positively correlated with the number of platform crossings $(r=0.611, P<0.05)$, time spent in the target quadrant ( $\%$ of total) $(r=0.652, P<0.05)$, time spent in the open arm $(r=0.810, P<0.01)$ and time spent in the central area (\% of total) $(r=0.732, P<0.01)$. Ktrans of the hippocampus was negatively correlated with the number of platform crossings ( $r=-0.724, P<0.01$ ), time spent in the target quadrant (\% of total) $(r=-0.700, P<0.05)$, time spent in the open $\operatorname{arm}(r=-0.634, P<0.05)$ and time spent in the central area (\% of total) $(r=-0.666, P<0.05)$.

\section{Nissl Staining}

$\mathrm{H}$ group rats showed significant morphological changes in their neurons in the CA1, CA3 and DG areas of the hippocampus. After 8 months of plateau hypoxia exposure, scattered, small, irregularly shaped and shrinking neurons and nuclear pyknosis were observed in the CA1, CA3 and DG areas of the hippocampus of $\mathrm{H}$ group rats (Fig. 8b).

\section{FJB-Staining}

FJB-staining revealed neurodegeneration of the hippocampus in the $\mathrm{H}$ and $\mathrm{P}$ group rats (Fig. 8c). $\mathrm{H}$ group rats showed a significant increase in FJB-positive cells in the CA1, CA3, and DG regions of the hippocampus compared with $P$ group rats $(P<0.001)$ (Fig. $8 \mathrm{~d})$.

\section{Immunofluorescent Examination of Iba-1}

$\mathrm{H}$ group rats, compared with $\mathrm{P}$ group rats, showed a significant increase in their microglial cell counts in the CA1 $(19.20 \pm 3.71$ vs. $11.80 \pm 1.72, P=0.0068)$, CA3 $(30.33 \pm 4.11$ vs. $9.67 \pm 2.56, P<0.001)$ and DG $(15.00 \pm 2.83$ vs. $10.00 \pm 2.00, P=0.0203)$ regions of the hippocampus (Fig. 9a and b).

\section{Immunofluorescent Examination of GFAP}

Compared with the $\mathrm{P}$ group rats, the $\mathrm{H}$ group rats showed a significant increase in astrocyte cell counts in the CA1 $(48.17 \pm 5.15$ vs. $37.17 \pm 3.80, P=0.0032)$, CA3 $(70.33 \pm 9.66$ vs. $60.17 \pm 2.67, P=0.0476)$ and DG $(61.00 \pm 11.43$ vs. $47.67 \pm 4.85, P=0.0373)$ regions of the hippocampus (Fig. 9c and d).

\section{Hippocampal Dendritic Complexity and Dendritic Spine Densities}

Compared with the control rats, the $\mathrm{H}$ group rats showed a decrease in the number of dendritic spines in the 


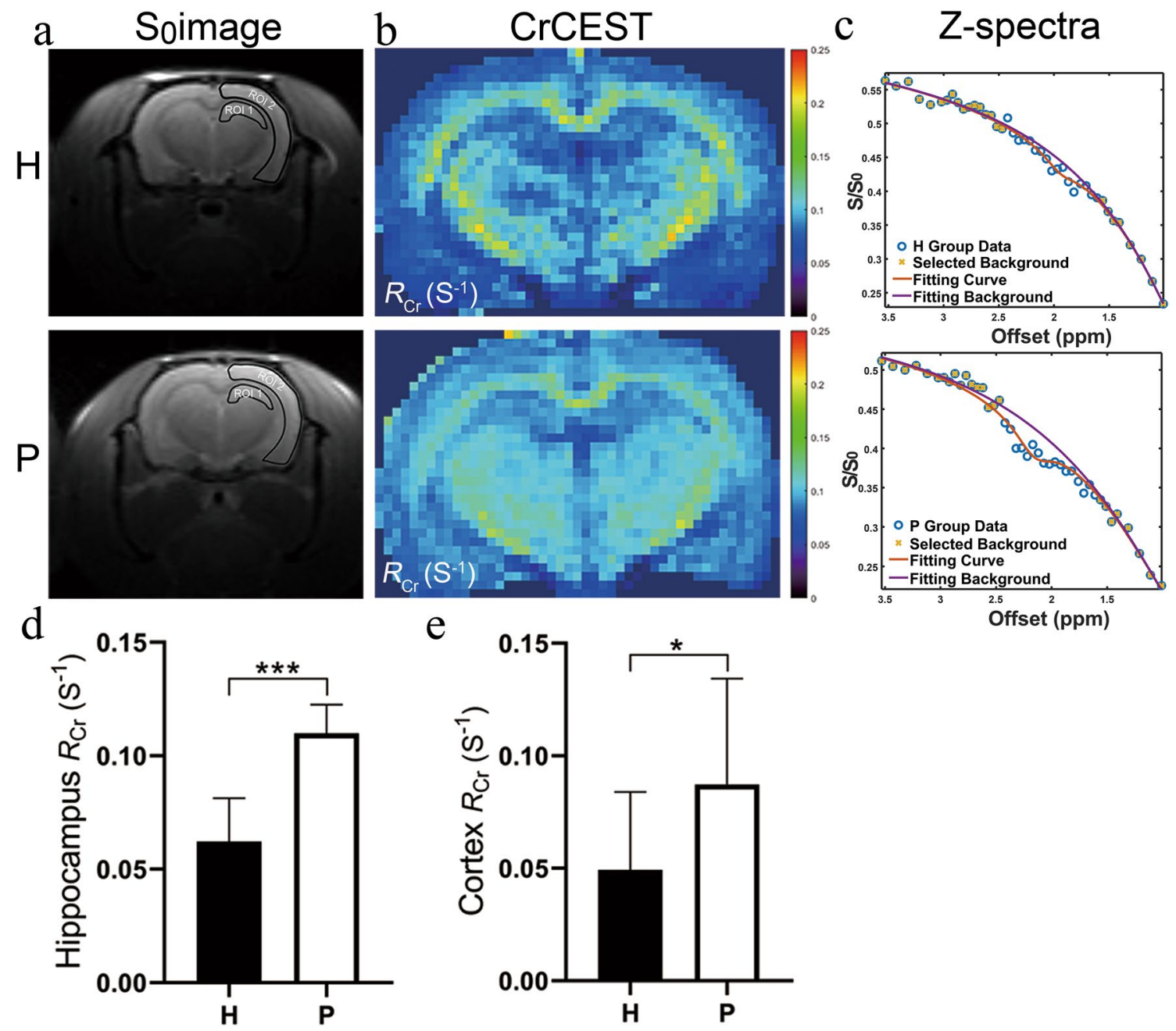

Fig. 5 Representative CrCEST in the hippocampus and the cortex of $\mathrm{H}$ and $\mathrm{P}$ group rats. Typical $\mathrm{S}_{0}$ images (a), cortical CrCEST maps (b), CrCEST Z-spectra (c) for $\mathrm{H}$ and $\mathrm{P}$ group rats. Both CrCEST Z-spectra and CrCEST maps $\left(R_{\mathrm{Cr}}\right)$ of $\mathrm{H}$ group rats show a clear signal reduction compared with $\mathrm{P}$ group rats. The ROIs used to extract regional values are indicated in (a). The Z-spectra in $\mathrm{c}$ are from the hip-

hippocampal CA1, CA3, and DG subregions and in the cortical region $(P<0.05)$ (Fig. 10a and b). The Sholl plots showed that dendritic intersections were distributed at an increasing distance from the center of the cell body (Fig. 10c). Repeated measurements with ANOVA of the number of intersections across the distances from the center of the cell body indicate a significant effect for the group $[F(1$, $94)=7.218 ; P=0.009$ in the CA1 region, $F(1,94)=5.671$; $P=0.019$ in the CA3 region, $F(1,94)=5.350 ; P=0.023$ in the DG region, and $F(1,94)=9.931 ; P=0.002$ in the cortical region], with a significant interaction between group and distance from the cell soma $[F(9,846)=4.151 ; P<0.0001$ in the CA1 region, $F(9,846)=4.148 ; P<0.0001$ in the CA3 region, $F(9,846)=1.205 ; P=0.029$ in the $\mathrm{DG}$ region, and $F(9,846)=2.068 ; P=0.030$ in the cortical region]. Sidak's pocampus (ROI 1$)$. Data are expressed as the mean \pm SD $(n=12$ per group). $H$ plateau hypoxia, $P$ plain, $R O I$ region of interest. $* P<0.05$, $* * * P<0.001$. Bar graphs show differences in the CrCEST apparent relaxation rates in the rotating frame $\left(R_{C r}\right)$ for hippocampus $(\mathbf{d})$ and cortex (e) between the $\mathrm{H}$ and $\mathrm{P}$ group rats

multiple comparisons show that compared with the control $\mathrm{P}$ group rats, $\mathrm{H}$ group rats had fewer Sholl intersections within 10-50 $\mu \mathrm{m}$ from the soma in the CA1 region, fewer Sholl intersections within $10-50 \mu \mathrm{m}$ from the soma in the CA3 region, fewer Sholl intersections in $100 \mu \mathrm{m}$ from the soma in the DG region, and fewer Sholl intersections within $10-40 \mu \mathrm{m}$ from the soma in the cortex regions (Fig. 10d).

\section{TUNEL Stain}

TUNEL-positive cells were observed in the hippocampal CA1, CA3 and DG subregions (Fig. 11a). A considerable number of TUNEL-positive neurons appeared in the hippocampal CA1, CA3, and DG of $\mathrm{H}$ group rats compared with that of $\mathrm{P}$ group rats $(P<0.01)$ (Fig. 11b). 

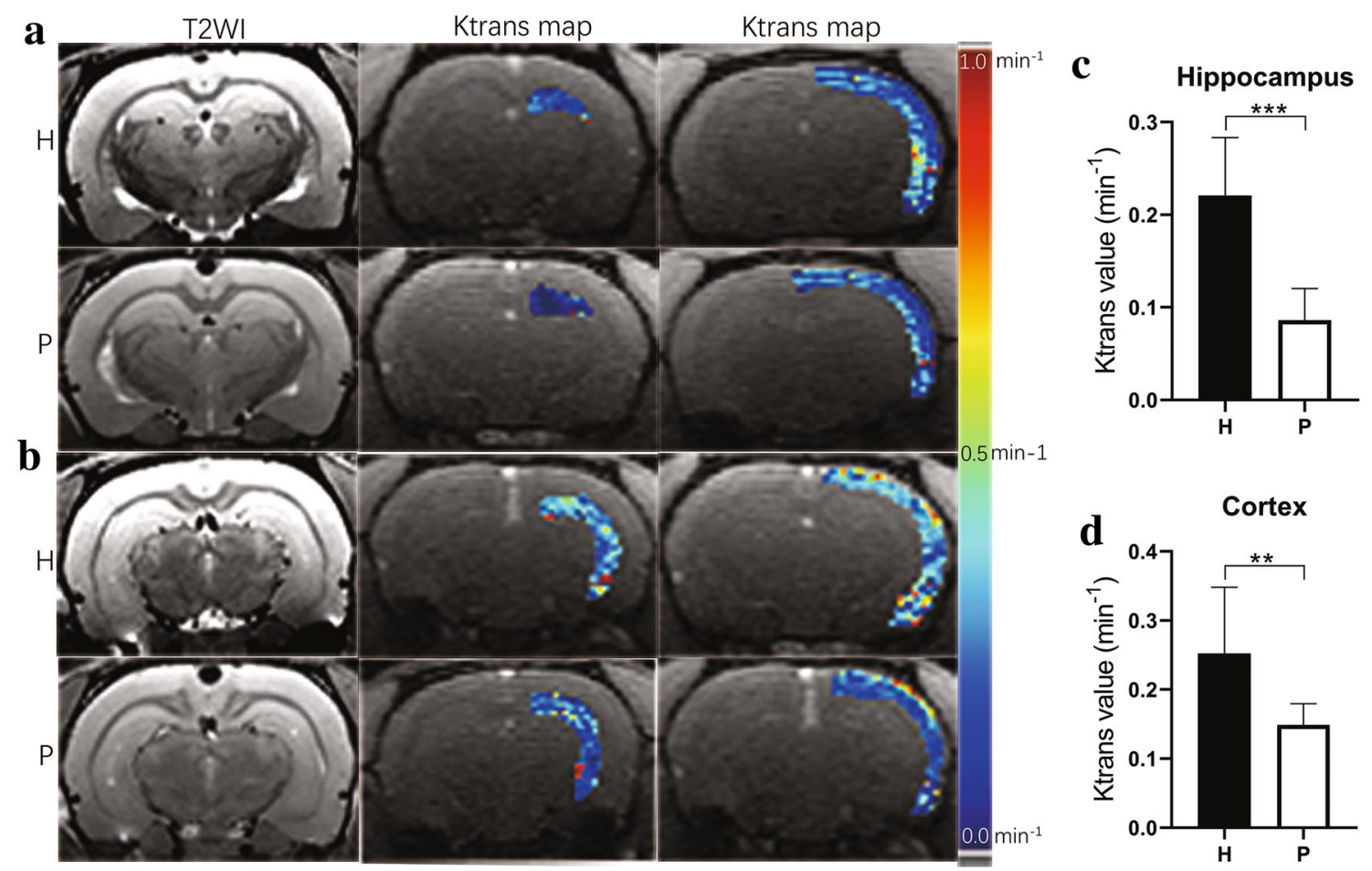

Fig. 6 BBB permeability increase in the hippocampus and ipsilateral cortex of $\mathrm{H}$ group rats. a Hippocampus and cortex of $\mathrm{H}$ and $\mathrm{P}$ group rats at $-3.60 \mathrm{~mm}$ relative to the anterior commissure (slice 1). b Hippocampus and cortex of $\mathrm{H}$ and $\mathrm{P}$ group rats at $-5.60 \mathrm{~mm}$ relative to the anterior commissure (slice 2). Left column shows hippocampus and cortex on T2WI. Middle column shows Ktrans maps of hippocampus. Right column shows Ktrans maps of cortex. Color-coded

\section{TEM Results}

$\mathrm{H}$ group rats showed neuronal apoptosis in four regions, viz., CA1, CA3, and DG, in the hippocampus and the cortex (Fig. 12). Specifically, apoptotic death was characterized by increased electron density, nuclear chromatin condensation, shrinkage of the nuclear membrane and perinuclear organelle vacuolization, which was found in the neurons of $H$ group rats. Additionally, the neurons and axons in the $\mathrm{H}$ group rats were mitochondrially swollen. The peripheral space in the vessels of the hippocampus of $\mathrm{H}$ group rats was widened. In contrast, no obvious ultrastructural abnormalities with neuronal cells and vessels were found in the above regions of the $\mathrm{P}$ group rats.

\section{Discussion}

In the present study, we used multimodal MR imaging to detect morphological, molecular and microvascular alterations in the hippocampus in a rat model of chronic exposure
Ktrans ranged between 0.0 and $1.0 \mathrm{~min}^{-1}$, with dark blue indicating $0.0 \mathrm{~min}^{-1}$, green indicating $0.5 \mathrm{~min}^{-1}$, and red indicating $1 \mathrm{~min}^{-1}$. c Ktrans of the right hippocampus of $\mathrm{H}$ and $\mathrm{P}$ group rats. d Ktrans of the right cortex of $\mathrm{H}$ and $\mathrm{P}$ group rats. Data are expressed as the mean $\pm \mathrm{SD}(\mathrm{n}=12$ per group). $H$ plateau hypoxia, $P$ plain, $B B B$ blood-brain barrier, Ktrans volume transfer constant. ${ }^{* *} P<0.01$, $* * * P<0.001$

to a hypoxic plateau environment. The results of the study show reduced rGMV, lower CrCEST contrast and higher transport coefficient Ktrans in the hippocampus of $\mathrm{H}$ group rats compared with that of the control, $\mathrm{P}$ group rats. Further histopathological examinations revealed neural degeneration and apoptosis, proliferation of glial cells and decreased spine density in the hippocampus of $\mathrm{H}$ group rats. Moreover, correlation analysis showed that quantitative MRI parameters of the hippocampus were associated with the behavioral performance of $\mathrm{H}$ group rats. These findings suggest that chronic exposure to a hypoxic plateau environment might adversely affect the gray matter, energy metabolism and microvascular permeability of the hippocampus of rats and thus contribute to deficits in affective and cognitive functions of $\mathrm{H}$ group rats.

Prior VBM studies have revealed that high-altitude residents with chronic exposure to hypobaric hypoxia showed significant structural modifications in the brain, which include a decrease in the regional cortical gray matter accompanied by changes in the white matter, which may underlie a cerebral adaptation to hypoxic exposure [13, 42, 

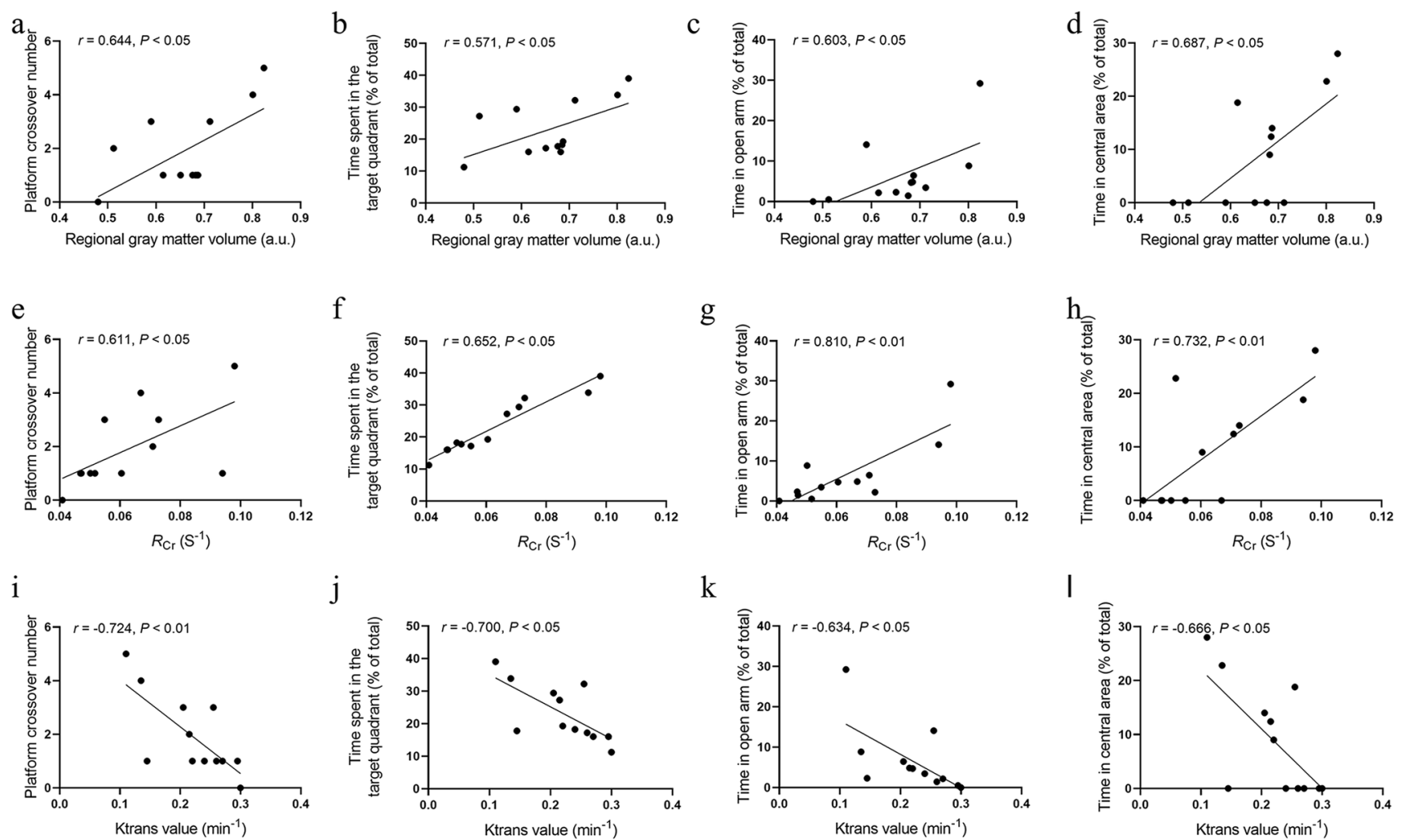

$\mathrm{k}$
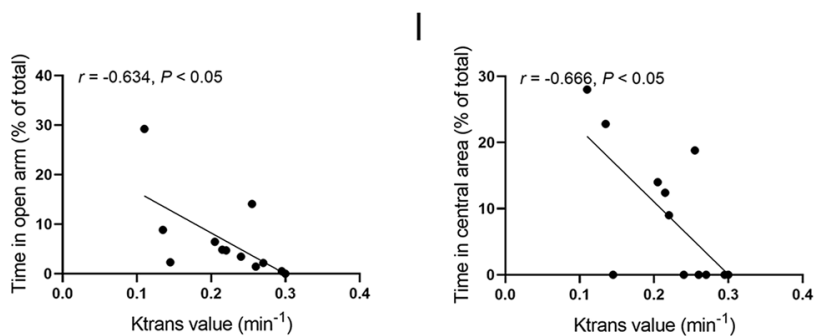

Fig. 7 Correlation analysis results. Regional gray matter volume (rGMV) of the hippocampus was positively correlated with the platform crossover numbers (a), time spent in the target quadrant (\% of total) (b), time in the open arm (c), and time in central area (\% of total) (d). The $R_{\mathrm{Cr}}$ of the hippocampus was positively correlated with the platform crossover numbers (e), time spent in the target quadrant

(\% of total) (f), time in the open arm (g), and time in the central area (\% of total) (h). Ktrans of the hippocampus was negatively correlated with the platform crossover numbers (i), time spent in the target quadrant (\% of total) $(\mathbf{j})$, time in the open arm (k), and time in the central area (\% of total) $(\mathbf{l})$

43]. However, there has been little direct investigation into morphological changes in the entire brains of $\mathrm{H}$ group rats after long-term exposure to plateau hypobaric hypoxia. VBM analysis in this study revealed gray matter atrophy in the hippocampus of rats exposed to plateau hypobaric hypoxia for 8 months. These findings are consistent with a previous report that the bilateral hippocampus was one of the first affected regions in rats after high-altitude exposure [6]. Therefore, VBM may provide qualitative indices of hypoxic plateau-induced brain damage to the regional volume or density reductions of the gray matter on the voxel scale. rGMV reduction was also found in the primary somatosensory cortex of $\mathrm{H}$ group rats compared with that of $\mathrm{P}$ group rats in this study.

It has been reported that many tissue properties, such as local cell count, spatial arrangement of cells and cell type composition, may affect the GMV [44]. Hypobaric hypoxia can induce neurodegeneration, which results in decreased dendritic arborization [45, 46]. Decreased dendritic arborization can cause GMV reduction [47]. Previous reports showed that there was remarkable neuron loss after hypoxic

challenge in the hippocampus [48]. Reduced rGMV, as demonstrated by structural MR imaging, can be used as a surrogate biomarker for neuronal losses [49, 50]. Thus, hypobaric hypoxia-induced changes in rGMV may be primarily related to neurodegeneration and death of cells in the hippocampus. Our correlation analysis revealed that plateau hypoxia exposure-induced learning deficits in the MWM test were directly associated with reduced rGMV in the hippocampus. Previous reports have come to similar conclusions that reduced rGMV in the hippocampus is associated with cognitive decline in patients with Parkinson's disease [51] and in animals with chronic epilepsy [52]. Thus, rGMV obtained with the VBM analysis method can be used as a reliable biomarker of cognitive impairment in rats and humans.

CrCEST can significantly enhance the sensitivity to low concentrations of $\mathrm{Cr}$ through exchangeable protonsis [53]. $\mathrm{Cr}$ reaches its peak at approximately $2 \mathrm{ppm}$ in the water saturation spectrum (Z-spectrum), whereas the effect of CrCEST depends linearly on $\mathrm{Cr}$ concentration in the physiological $\mathrm{pH}$ range [35]. The guanidinium protons in $\mathrm{Cr}$ and the amide and guanidine protons in phosphocreatine $(\mathrm{PCr})$ are 


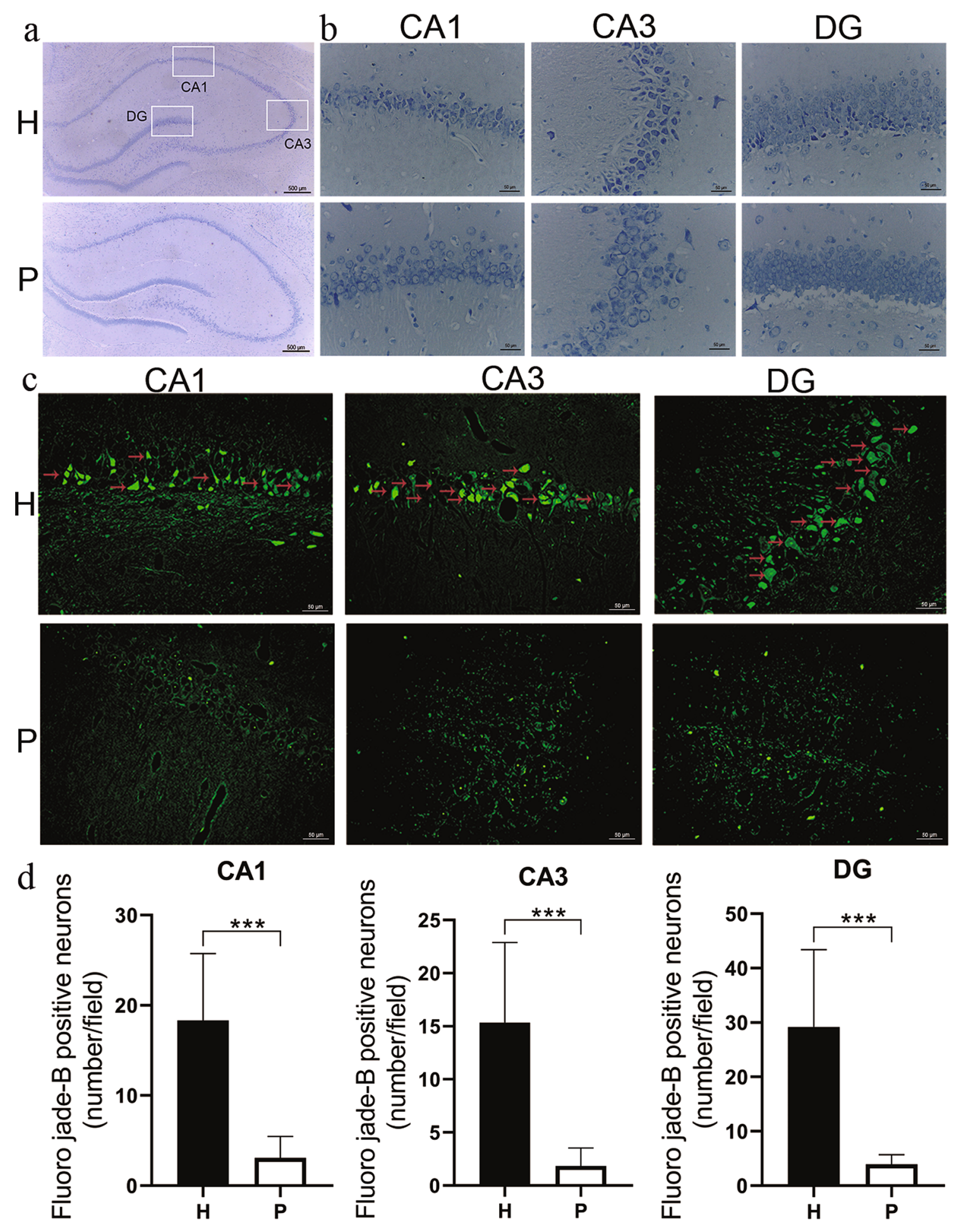

Fig. 8 Effect of plateau hypoxia exposure on morphological changes in hippocampal neurons of rats. Representative scheme of the CA1, CA3, and DG subregions considered in the analysis (a). In the hippocampus of $\mathrm{H}$ group rats, pyknotic, small sized, dense and irregularly shaped cells were observed by Nissl staining (b). Degenerative neurons (red arrow) were labeled by Fluoro Jade-B staining (c). The graph represents the morphometric results of Fluoro Jade-B staining (d). Scale bar $=500 \mu \mathrm{m}$ in (a) and $50 \mu \mathrm{m}$ in (b) and (c). Data are expressed as the mean $\pm \mathrm{SD}$ ( $\mathrm{n}=6$ per group). $H$ plateau hypoxia, $P$ plain. $* * * P<0.001$ 

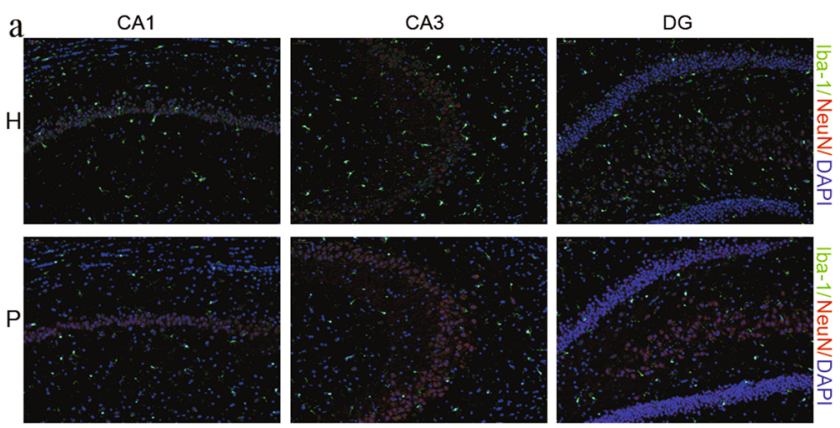

b
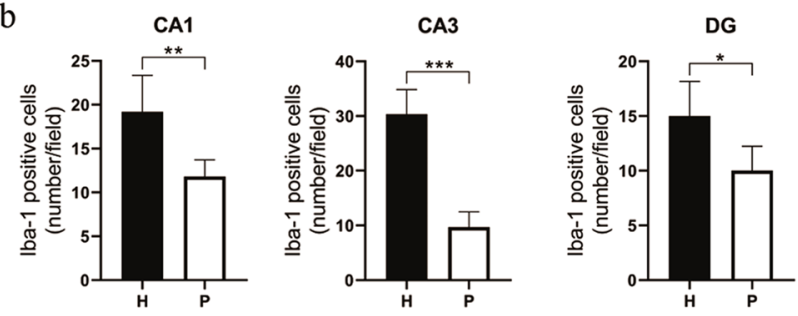

Fig. 9 Iba- 1 and GFAP increase in the hippocampus of $\mathrm{H}$ group rats. Representative immunofluorescence staining of microglia (Iba-1-positive cells) in the CA1, CA3, and DG areas (a). Bar graphs showing Iba-1-positive cell counts in the CA1, CA3, and DG areas (b). Representative immunofluorescence staining of astrocytes (GFAP-positive cells) in the CA1, CA3, and DG areas (c). Bar graphs showing

exchangeable with water. $\mathrm{PCr}$ produces adenosine triphosphate, which is a common currency of bioenergy, by losing phosphorus. As this process is reversible, $\mathrm{Cr}$ can be phosphorylated to form $\mathrm{PCr}$, which is often used as an energy reserve to quickly produce large amounts of ATP. Therefore, it is essential to detect $\mathrm{Cr}$ and $\mathrm{PCr}$ for the detection of many energy-related diseases. Previous CrCEST studies of the brain [54], muscle [55] and myocardium [56] have provided promising data and evidence supporting the feasibility of CrCEST to freely detect $\mathrm{Cr}$ at millimolar physiological concentrations. Recent studies have reported a significant linear correlation between the testicular CrCEST effect and testicular ischemia time [47] and spermatogenesis [48]. Although $1 \mathrm{H}$ magnetic resonance spectroscopy (MRS) and 31P MRS can also detect $\mathrm{Cr}$ and $\mathrm{PCr}$, their low detection sensitivity, poor spatial resolution and longer acquisition duration make it difficult for MRI imaging to detect free $\mathrm{Cr}$ signals with satisfactory precision [57]. In addition, most MRI instruments today are still not equipped with phosphorus coils specific to 31P MRS.

Cr concentration, measured by CrCEST in our study, could differentiate $\mathrm{H}$ group rats and $\mathrm{P}$ group rats and was significantly and positively correlated with neurological deficits. As $\mathrm{Cr}$ can be neuroprotective [58], its loss may be viewed as a reflection of neuronal cell death $[59,60]$. Previous studies revealed that altered brain bioenergetics were observed in subjects recruited in cities of moderately
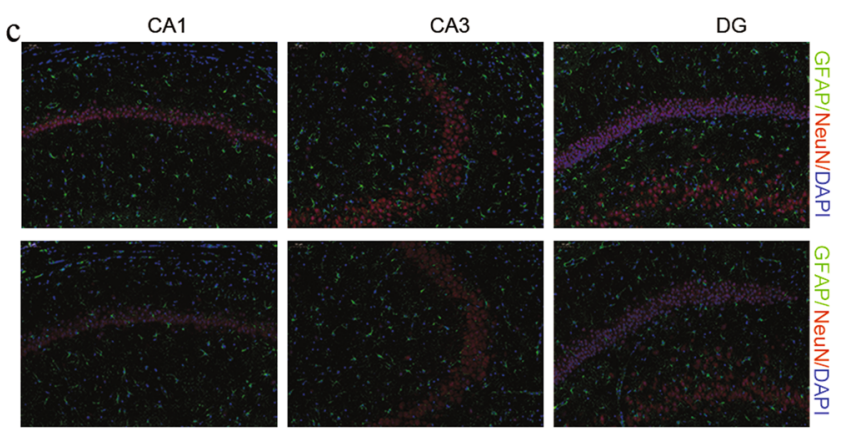

d
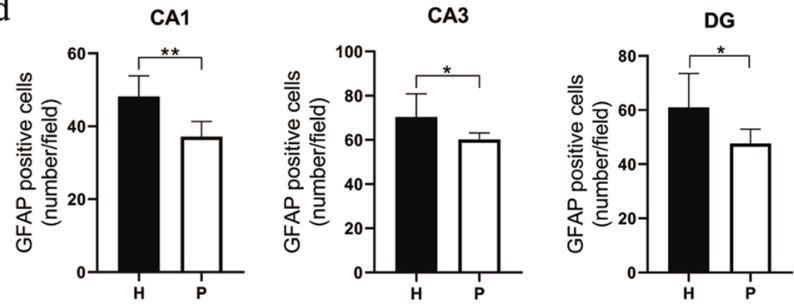

GFAP-positive cell counts in the CA1, CA3, and DG areas (d). Data are expressed as the mean $\pm \mathrm{SD}(\mathrm{n}=6$ per group). $H$ plateau hypoxia, $P$ plain, $N e u N$ neuronal nuclei, $I b a-1$ ionized calcium binding adaptor molecule-1, and GFAP glial fibrillary acidic protein. ${ }^{*} P<0.05$, $* * P<0.01, * * * P<0.001$

elevated altitude [61]. Loss and depletion of energy metabolites in the hippocampus after chronic plateau hypoxia exposure might possibly be useful biomarkers of cognitive impairment in animals and humans.

A recent study has suggested that CrCEST is highly sensitive to subtle $\mathrm{pH}$ variations in the brains of patients with early Alzheimer's disease [27]. It has been reported that neuroinflammation can cause intracellular cerebral $\mathrm{pH}$ reduction $[62,63]$. Previous studies have revealed that high altitude is related to changes in the intracellular $\mathrm{pH}$ and inorganic phosphate levels of the brain [64-66]. However, due to the lack of a method to test intracellular $\mathrm{pH}$, we cannot rule out the likelihood that the decreased CrCEST contrast is probably related to a decreased intracellular $\mathrm{pH}$ rather than the loss of $\mathrm{Cr}$ concentration or a combination of both. Therefore, it has to be elucidated in further studies how or whether neuroinflammation-triggered reduction of intracellular $\mathrm{pH}$ is related to the decrease in $\mathrm{pH}$-dependent CrCEST contrast after chronic exposure to plateau hypoxia.

BBB integrity is usually maintained by three main elements, including brain microvascular endothelial cells, astrocytic end-feet and pericytes [67]. Brain microvascular endothelial cells play an essential role in maintaining the normal functions of the BBB primarily through the presence of tight junction proteins. Although dyes such as Evans blue are commonly used as biomarkers of brain barrier integrity, Evans blue is not satisfactory enough for the study of BBB 

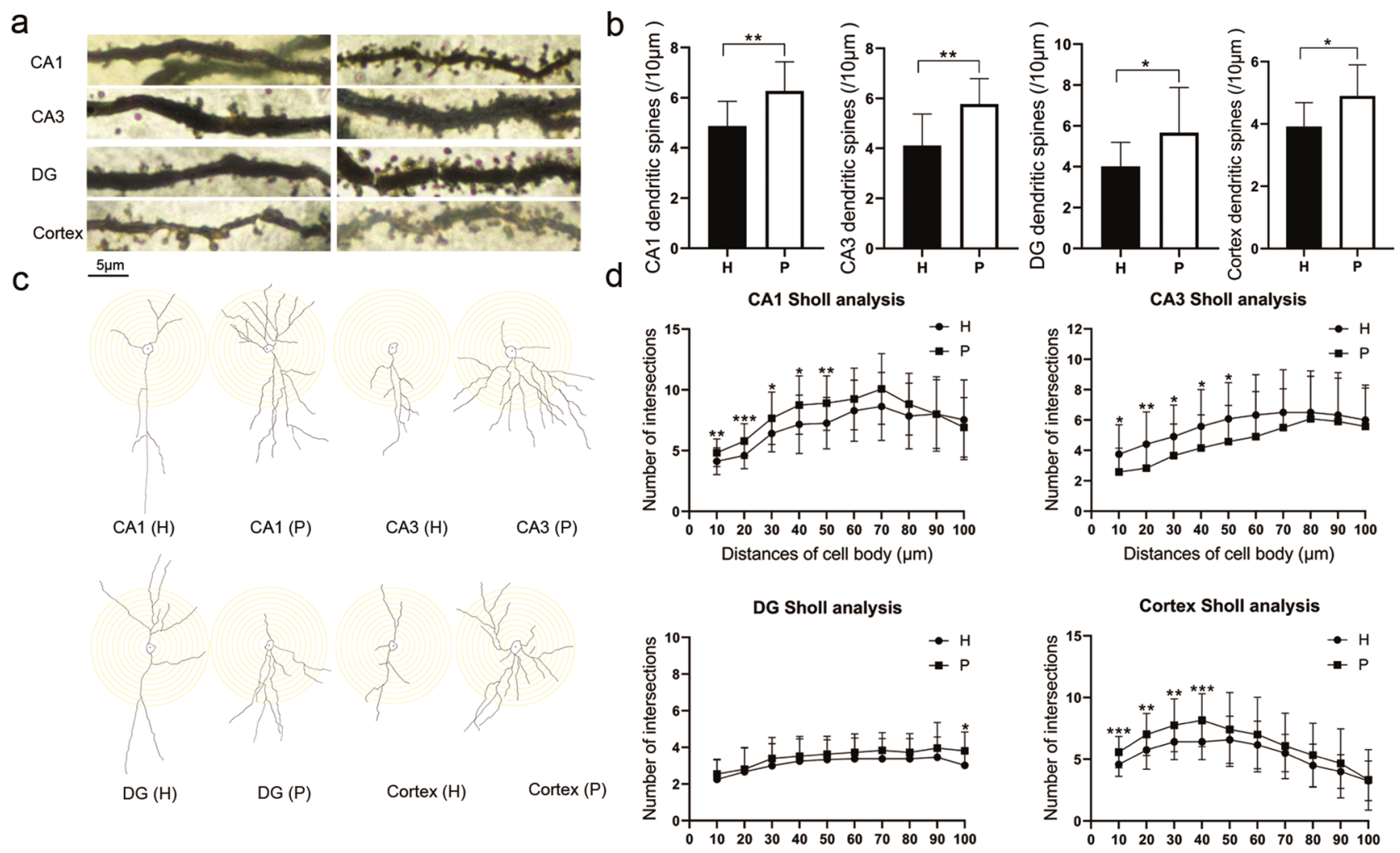

Fig. 10 Modified Golgi-Cox staining showing the differences in hippocampal and cortical neurons between the $\mathrm{H}$ and $\mathrm{P}$ group rats. Neurons of $\mathrm{H}$ and $\mathrm{P}$ group rats were imaged under a $100 \times$ lens (a). The $\mathrm{H}$ group rats showed a reduced number of dendrites compared with the $\mathrm{P}$ group rats $(\mathbf{b})$. Schematic photomicrographs of neurons $(20 \times)$ with the allocation of dendrites between repeated $10 \mu \mathrm{m}$-spaced concentric rings (c). The $\mathrm{H}$ group rats showed a reduced number of neuronal intersections in the hippocampus and cortex compared with the $\mathrm{P}$ group rats $(\mathbf{d})$. Scale bar $=5 \mu \mathrm{m}$. Data are expressed as the mean $\pm \mathrm{SD}$ (n=6 per group). $H$ plateau hypoxia, $P$ plain. $* P<0.05$, $* * P<0.01$ dysfunction because it is not usable under most circumstances [68].

As DCE-MRI can specifically evaluate BBB integrity by measuring $\mathrm{BBB}$ leakage to quantify the extent of contrast extravasation to the brain, it has thus paved the way for further studies of the microcirculation in preclinical and clinical settings [69]. In addition to measuring BBB disruption in tumors, multiple sclerosis and acute ischemic stroke, DCE-MRI has recently been used to measure more subtle and chronic BBB disruptions, as observed in neurodegenerative diseases such as Alzheimer's disease and Parkinson's disease [70, 71].

Previous studies have reported that chronic exposure to hypobaric hypoxia causes apparent anatomical variations in the BBB [72, 73] and diminishes the integrity of the BBB [74, 75]. Ktrans, an important metric of DCE-MRI and a constant of transfer, is widely used to evaluate BBB permeability in vivo. Ktrans is also a complicated combination of the tissue perfusion flow rate with permeability-surface area per volume of tissue. In the present study, we discovered that compared with $\mathrm{P}$ group rats, $\mathrm{H}$ group rats had dramatically higher Ktrans values estimated by the extended Tofts model in the hippocampus and the cortex, which is indicative of an increased BBB permeability in these animals. A perivascular lake formed by fluid extravasation was observed under an electronic microscope, which might be characteristic of vasogenic edema [73]. Therefore, based on a DCE-MRI study, the results of our study indicate that chronic exposure to plateau hypoxia may increase BBB permeability in the hippocampus and thus compromise the integrity of the BBB. Disruptions of the BBB in the hippocampus have been reported to probably contribute to cognitive impairment [76, 77]. This may plausibly provide further data that support the claim that cognitive impairment is caused by high-altitude hypoxia.

High-altitude exposure may induce chronic neuroinflammation and microglial activation [74, 78], which may damage the BBB. With chronic exposure of $\mathrm{H}$ group rats to hypobaric hypoxia, proinflammatory cytokines in their plasma and brain can be upregulated [79], which might be primarily responsible for BBB disruption [80]. In view of this, we suggest that quantitative parameters derived from DCE-MRI can be used as effective biomarkers of BBB damage $[36,81]$ associated with neuroinflammation [82]. 

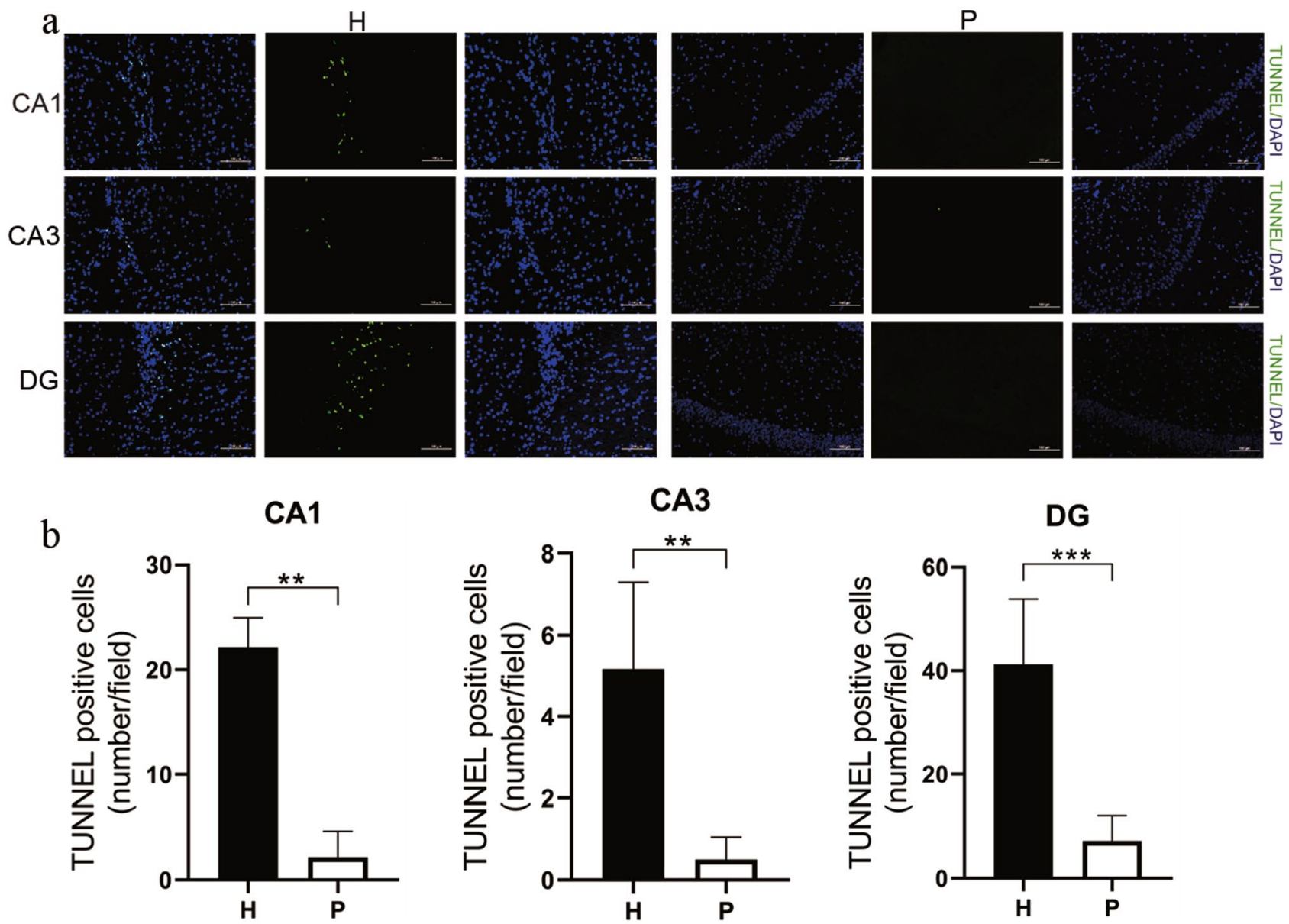

Fig. 11 TUNEL immunofluorescent staining in the CA1, CA3, and DG areas (a). Bar graphs showing TUNEL-positive cell counts in the CA1, CA3, and DG areas. Scale bar $=100 \mu \mathrm{m}$. Data are expressed as

the mean $\pm \mathrm{SD}$ (n=6 per group). $H$ plateau hypoxia, $P$ plain, $T U N E L$ TdT-mediated dUTP Nick-end Labeling. $* * * P<0.001$

After 8 months of exposure to plateau hypoxia, oxidative stress, chronic neuroinflammation, neuronal degeneration and apoptosis were observed in the hippocampal tissues of $\mathrm{H}$ group rats, which may be indicative of continued hypoxic brain damage. In addition, increased proinflammatory cytokine levels and white blood cell counts in the blood of $\mathrm{H}$ group rats were also observed, which may be indicative of systemic inflammation after long-term exposure to a highaltitude environment. Chronic neuroinflammation may cause alterations to energy metabolism and BBB permeability in the hippocampus and decrease hippocampal spine density. There has been considerable evidence that neuroinflammatory responses and oxidative damage can affect hippocampal neurons, segue into measurable gray matter loss and eventually lead to cognitive disorders over time [83]. Therefore, we suggest that long-term plateau hypoxia exposure may further wear out the brain structure and physical and pathophysiological functions of $\mathrm{H}$ group rats.

Several limitations in this study should be acknowledged. First, it utilized only single-slice CrCEST and double-slice DCE imaging in the gross hippocampus region, whereas multiple cross-sectional CrCEST and DCE imaging analyses are needed to further validate the conclusion of this study. Second, CEST acquisitions were performed under anesthesia with isoflurane, whose effect on Cr levels in the hippocampus of the rats is not to be neglected [84]. Third, we chose male rats as the experimental model to exclude the influencing factors of fluctuations in reproductive hormones, as estrogen appears to affect sensitivity to hypoxia-induced hippocampal damage in female rats [85]. In addition, female rats were not used in this study to reduce variability due to endocrine cycles. Thus, we used only male rats for behavioral consistency. Finally, we used a Sprague-Dawley male animal model of chronic plateau hypoxia that exhibited genetic variability in a plateau environment after prolonged hypoxia exposure, whereas other animals, such as canines 


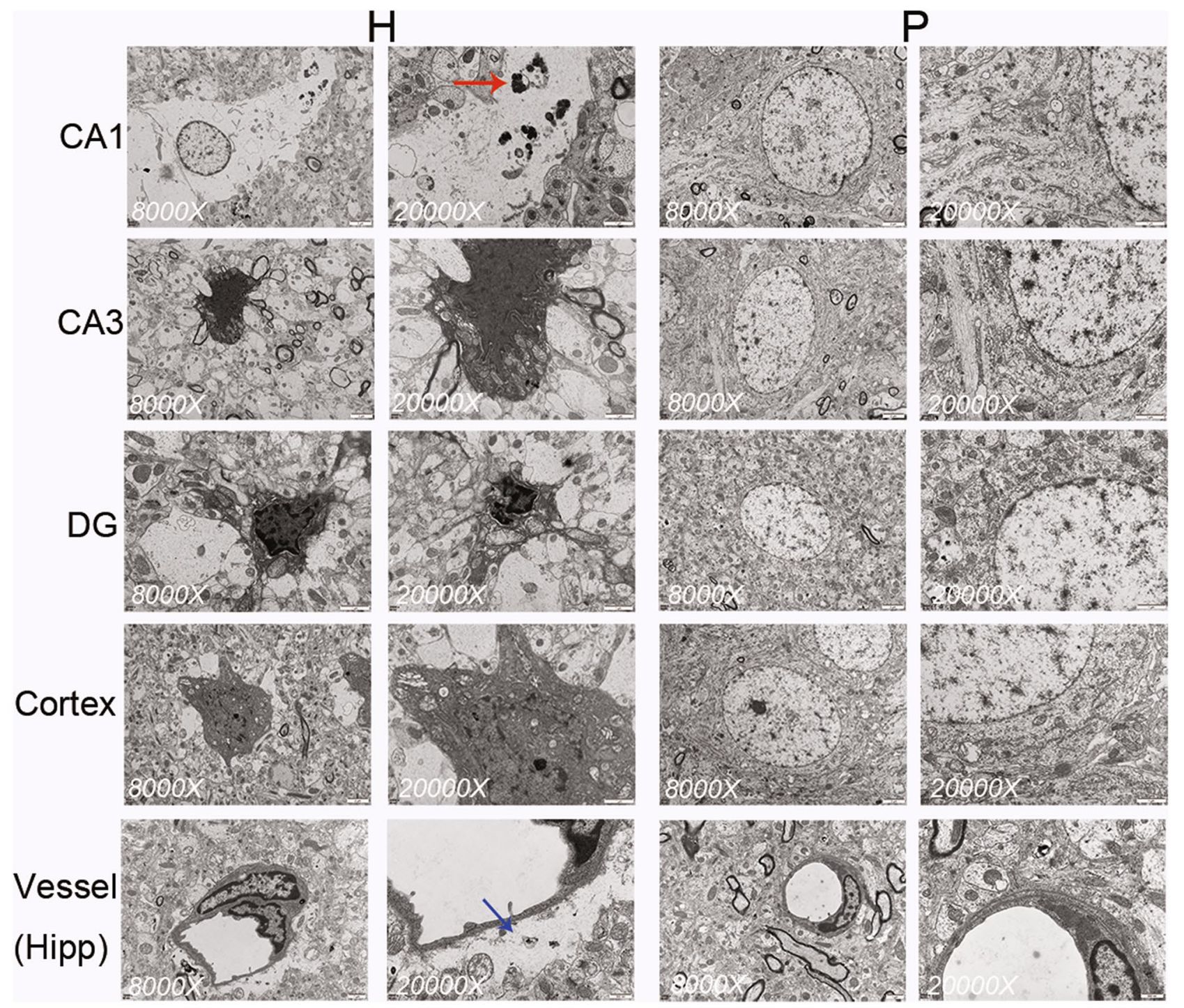

Fig. 12 Plateau hypoxia-induced neuroapoptosis and autophagy (as indicated with red arrows) in the hippocampus and cortex of rats ( $\mathrm{n}=3$ per group). Widespread perivascular spaces were seen in the hippocampus (blue arrows). Scale bar $=100 \mu \mathrm{m}$

and nonhuman primates or mice, were not utilized but should be used in future studies.

\section{Conclusions}

Our study demonstrated hippocampal damage in a rat model after chronic exposure for 8 months to a hypoxic plateau environment. Multimodal noninvasive MR imaging (VBM, CrCEST, and DCE), by measuring alterations in rGMV, CrCEST contrast and Ktrans values, revealed hippocampal atrophy, decreased $\mathrm{Cr}$ concentration and increased BBB permeability in $\mathrm{H}$ group rats compared with control rats. Further correlation analysis shows that the altered MR imaging parameters in the hippocampus of $\mathrm{H}$ group rats were associated with cognitive dysfunctions. In summary, these findings suggest that multimodal MR imaging techniques can be used as effective means to detect plateau hypoxiainduced hippocampal damage and provide potential biomarkers for understanding the mechanism underlying plateau hypoxia-mediated affective and cognitive dysfunctions.

Supplementary Information The online version contains supplementary material available at https://doi.org/10.1007/s11064-021-03498-5.

Acknowledgements Thanks go to Duojie Dingda and Angwen Laji of the Qinghai Yushu People's Hospital, Yushu, China, for their assistance with their long-term animal feeding, water feeding and bedding change in the plateau animal laboratory. We would like to thank Xiaoting Chen from the Animal Experimental Center of West China Hospital, Chengdu, China, for her technical assistance in the animal experiments. 
Author Contributions FG conceptualized the project. FG and DZ designed the study and drafted the manuscript. DZ, BH, MZ, YW, RL, $\mathrm{LW}, \mathrm{YZ}$, and YL contributed to the literature research, data collection and analysis, and data interpretation. FG critically revised the manuscript. All the authors approved the final version of the manuscript.

Funding This study was supported by the National Natural Science Foundation of China (Grant No. 81930046, 81771800, and 81829003).

\section{Declarations}

Conflict of interest The authors declare that they have no conflict of interest to disclose.

Open Access This article is licensed under a Creative Commons Attribution 4.0 International License, which permits use, sharing, adaptation, distribution and reproduction in any medium or format, as long as you give appropriate credit to the original author(s) and the source, provide a link to the Creative Commons licence, and indicate if changes were made. The images or other third party material in this article are included in the article's Creative Commons licence, unless indicated otherwise in a credit line to the material. If material is not included in the article's Creative Commons licence and your intended use is not permitted by statutory regulation or exceeds the permitted use, you will need to obtain permission directly from the copyright holder. To view a copy of this licence, visit http://creativecommons.org/licenses/by/4.0/.

\section{References}

1. Chen X, Zhang Q, Wang J, Liu J, Zhang W, Qi S, Xu H, Li C, Zhang J, Zhao $\mathrm{H}$ et al (2017) Cognitive and neuroimaging changes in healthy immigrants upon relocation to a high altitude: a panel study. Hum Brain Mapp 38(8):3865-3877

2. Sharma R, Cramer NP, Perry B, Adahman Z, Murphy EK, Xu X, Dardzinski BJ, Galdzicki Z, Perl DP, Dickstein DL et al (2019) Chronic exposure to high altitude: synaptic, astroglial and memory changes. Sci Rep 9(1):16406

3. Ji W, Zhang Y, Luo J, Wan Y, Liu J, Ge RL (2021) Memantine ameliorates cognitive impairment induced by exposure to chronic hypoxia environment at high altitude by inhibiting excitotoxicity. Life Sci 270:119012

4. O'Keefe J, Burgess N, Donnett JG, Jeffery KJ, Maguire EA (1998) Place cells, navigational accuracy, and the human hippocampus. Philos Trans R Soc Lond B Biol Sci 353(1373):1333-1340

5. Hota SK, Barhwal K, Baitharu I, Prasad D, Singh SB, Ilavazhagan G (2009) Bacopa monniera leaf extract ameliorates hypobaric hypoxia induced spatial memory impairment. Neurobiol Dis 34(1):23-39

6. Maiti P, Singh SB, Mallick B, Muthuraju S, Ilavazhagan G (2008) High altitude memory impairment is due to neuronal apoptosis in hippocampus, cortex and striatum. J Chem Neuroanat 36(3-4):227-238

7. Barhwal K, Das SK, Kumar A, Hota SK, Srivastava RB (2015) Insulin receptor A and Sirtuin 1 synergistically improve learning and spatial memory following chronic salidroside treatment during hypoxia. J Neurochem 135(2):332-346

8. Hota KB, Hota SK, Srivastava RB, Singh SB (2012) Neuroglobin regulates hypoxic response of neuronal cells through Hif- $1 \alpha$ - and Nrf2-mediated mechanism. J Cereb Blood Flow Metab Off J Int Soc Cereb Blood Flow Metab 32(6):1046-1060

9. Kushwah N, Jain V, Dheer A, Kumar R, Prasad D, Khan N (2018) Hypobaric hypoxia-induced learning and memory impairment: elucidating the role of small conductance $\mathrm{Ca}(2+)$-activated $\mathrm{K}(+)$ channels. Neuroscience 388:418-429

10. Barhwal K, Singh SB, Hota SK, Jayalakshmi K, Ilavazhagan G (2007) Acetyl-L-carnitine ameliorates hypobaric hypoxic impairment and spatial memory deficits in rats. Eur J Pharmacol 570(1-3):97-107

11. Bandopadhyay R, Singh T, Ghoneim MM, Alshehri S, Angelopoulou E, Paudel YN, Piperi C, Ahmad J, Alhakamy NA, Alfaleh MA et al (2021) Recent developments in diagnosis of epilepsy: scope of microRNA and technological advancements. Biology 10(11):1097

12. Ashburner J, Friston KJ (2005) Unified segmentation. Neuroimage 26(3):839-851

13. Yan X, Zhang J, Shi J, Gong Q, Weng X (2010) Cerebral and functional adaptation with chronic hypoxia exposure: a multi-modal MRI study. Brain Res 1348:21-29

14. Shukitt-Hale B, Kadar T, Marlowe BE, Stillman MJ, Galli RL, Levy A, Devine JA, Lieberman HR (1996) Morphological alterations in the hippocampus following hypobaric hypoxia. Hum Exp Toxicol 15(4):312-319

15. Chen L, Wei Z, Chan KWY, Cai S, Liu G, Lu H, Wong PC, van Zijl PCM, Li T, Xu J (2019) Protein aggregation linked to Alzheimer's disease revealed by saturation transfer MRI. Neuroimage 188:380-390

16. Wang R, Chen P, Shen Z, Lin G, Xiao G, Dai Z, Zhang B, Chen Y, Lai L, Zong X et al (2019) Brain amide proton transfer imaging of rat with Alzheimer's disease using saturation with frequency alternating RF irradiation method. Front Aging Neurosci 11:217

17. DeBrosse C, Nanga RPR, Wilson N, D'Aquilla K, Elliott M, Hariharan H, Yan F, Wade K, Nguyen S, Worsley D et al (2016) Muscle oxidative phosphorylation quantitation using creatine chemical exchange saturation transfer (CrCEST) MRI in mitochondrial disorders. JCI Insight 1(18):e88207

18. Kumar D, Nanga RPR, Thakuri D, Wilson N, Cember A, Martin ML, Zhu D, Shinohara RT, Qin Q, Hariharan H et al (2021) Recovery kinetics of creatine in mild plantar flexion exercise using 3D creatine CEST imaging at 7 Tesla. Magn Reson Med 85(2):802-817

19. Jing L, Shao J, Sun W, Lan T, Jia Z, Ma H, Wang H (2020) Protective effects of two novel nitronyl nitroxide radicals on heart failure induced by hypobaric hypoxia. Life Sci 248:116481

20. Li J, Qi Y, Liu H, Cui Y, Zhang L, Gong H, Li Y, Li L, Zhang Y (2013) Acute high-altitude hypoxic brain injury: identification of ten differential proteins. Neural Regen Res 8(31):2932-2941

21. Sourbron SP, Buckley DL (2013) Classic models for dynamic contrast-enhanced MRI. NMR Biomed 26(8):1004-1027

22. Cuenod CA, Balvay D (2013) Perfusion and vascular permeability: basic concepts and measurement in DCE-CT and DCE-MRI. Diagn Interv Imaging 94(12):1187-1204

23. Roach RC, Hackett PH (2001) Frontiers of hypoxia research: acute mountain sickness. J Exp Biol 204(Pt 18):3161-3170

24. Zhang $\mathrm{K}$, Zhao T, Huang $\mathrm{X}$, Liu ZH, Xiong L, Li MM, Wu LY, Zhao YQ, Zhu LL, Fan M (2009) Preinduction of HSP70 promotes hypoxic tolerance and facilitates acclimatization to acute hypobaric hypoxia in mouse brain. Cell Stress Chaperones 14(4):407-415

25. Nakanishi K, Tajima F, Nakata Y, Osada H, Tachibana S, Kawai T, Torikata C, Suga T, Takishima K, Aurues T et al (1999) Expression of endothelin-1 in rats developing hypobaric hypoxia-induced pulmonary hypertension. Lab Investig J Tech Methods Pathol 79(11):1347-1357

26. Vorhees CV, Williams MT (2006) Morris water maze: procedures for assessing spatial and related forms of learning and memory. Nat Protoc 1(2):848-858

27. Chen L, van Zijl PCM, Wei Z, Lu H, Duan W, Wong PC, Li T, $\mathrm{Xu} \mathrm{J}$ (2021) Early detection of Alzheimer's disease using creatine 
chemical exchange saturation transfer magnetic resonance imaging. NeuroImage 236:118071

28. Chen L, Wei Z, Cai S, Li Y, Liu G, Lu H, Weiss RG, van Zij1 PCM, Xu J (2019) High-resolution creatine mapping of mouse brain at $11.7 \mathrm{~T}$ using non-steady-state chemical exchange saturation transfer. NMR Biomed 32(11):e4168

29. Sargolzaei S, Cai Y, Wolahan SM, Gaonkar B, Sargolzaei A, Giza CC, Harris NG (2018) A comparative study of automatic approaches for preclinical MRI-based brain segmentation in the developing rat. Annu Int Conf IEEE Eng Med Biol Soc IEEE Eng Med Biol Soc Annu Int Conf 2018:652-655

30. Yushkevich PA, Piven J, Hazlett HC, Smith RG, Ho S, Gee JC, Gerig G (2006) User-guided 3D active contour segmentation of anatomical structures: significantly improved efficiency and reliability. Neuroimage 31(3):1116-1128

31. Sasaki K, Sumiyoshi A, Nonaka H, Kasahara Y, Ikeda K, Hall FS, Uhl GR, Watanabe M, Kawashima R, Sora I (2015) Specific regions display altered grey matter volume in $\mu$-opioid receptor knockout mice: MRI voxel-based morphometry. Br J Pharmacol 172(2):654-667

32. Barrière DA, Magalhães R, Novais A, Marques P, Selingue E, Geffroy F, Marques F, Cerqueira J, Sousa JC, Boumezbeur F et al (2019) The SIGMA rat brain templates and atlases for multimodal MRI data analysis and visualization. Nat Commun 10(1):5699

33. Sumiyoshi A, Nonaka H, Kawashima R (2017) Sexual differentiation of the adolescent rat brain: a longitudinal voxel-based morphometry study. Neurosci Lett 642:168-173

34. Chen L, Barker PB, Weiss RG, van Zijl PCM, Xu J (2019) Creatine and phosphocreatine mapping of mouse skeletal muscle by a polynomial and Lorentzian line-shape fitting CEST method. Magn Reson Med 81(1):69-78

35. Chen L, Zeng H, Xu X, Yadav NN, Cai S, Puts NA, Barker PB, Li T, Weiss RG, van Zijl PCM et al (2017) Investigation of the contribution of total creatine to the CEST Z-spectrum of brain using a knockout mouse model. NMR Biomed 30(12):e3834

36. Xu Z, Zeng W, Sun J, Chen W, Zhang R, Yang Z, Yao Z, Wang L, Song L, Chen Y et al (2017) The quantification of blood-brain barrier disruption using dynamic contrast-enhanced magnetic resonance imaging in aging rhesus monkeys with spontaneous type 2 diabetes mellitus. Neuroimage 158:480-487

37. Singh T, Joshi S, Williamson JM, Kapur J (2020) Neocortical injury-induced status epilepticus. Epilepsia 61(12):2811-2824

38. Scallet AC, Schmued LC, Slikker W Jr., Grunberg N, Faustino PJ, Davis H, Lester D, Pine PS, Sistare F, Hanig JP (2004) Developmental neurotoxicity of ketamine: morphometric confirmation, exposure parameters, and multiple fluorescent labeling of apoptotic neurons. Toxicol Sci Off J Soc Toxicol 81(2):364-370

39. Hara H, Onodera H, Kogure K, Akaike N (1990) The regional difference of neuronal susceptibility in the dentate gyrus to hypoxia. Neurosci Lett 115(2-3):189-194

40. Narayanan SN, Jetti R, Gorantla VR, Kumar RS, Nayak S, Bhat PG (2014) Appraisal of the effect of brain impregnation duration on neuronal staining and morphology in a modified Golgi-Cox method. J Neurosci Methods 235:193-207

41. Zhang H, Kuang H, Luo Y, Liu S, Meng L, Pang Q, Fan R (2019) Low-dose bisphenol A exposure impairs learning and memory ability with alterations of neuromorphology and neurotransmitters in rats. Sci Total Environ 697:134036

42. Zhang J, Zhang H, Li J, Chen J, Han Q, Lin J, Yang T, Fan M (2013) Adaptive modulation of adult brain gray and white matter to high altitude: structural MRI studies. PloS one 8(7):e68621

43. Zhang J, Yan X, Shi J, Gong Q, Weng X, Liu Y (2010) Structural modifications of the brain in acclimatization to high-altitude. PloS one 5(7): 11449

44. Asan L, Falfán-Melgoza C, Beretta CA, Sack M, Zheng L, WeberFahr W, Kuner T, Knabbe J (2021) Cellular correlates of gray matter volume changes in magnetic resonance morphometry identified by two-photon microscopy. Sci Rep 11(1):4234

45. Titus AD, Shankaranarayana Rao BS, Harsha HN, Ramkumar K, Srikumar BN, Singh SB, Chattarji S, Raju TR (2007) Hypobaric hypoxia-induced dendritic atrophy of hippocampal neurons is associated with cognitive impairment in adult rats. Neuroscience 145(1):265-278

46. Maiti P, Muthuraju S, Ilavazhagan G, Singh SB (2008) Hypobaric hypoxia induces dendritic plasticity in cortical and hippocampal pyramidal neurons in rat brain. Behav Brain Res 189(2):233-243

47. Alexander GE, Lin L, Yoshimaru ES, Bharadwaj PK, Bergfield KL, Hoang LT, Chawla MK, Chen K, Moeller JR, Barnes CA et al (2020) Age-related regional network covariance of magnetic resonance imaging gray matter in the rat. Front Aging Neurosci $12: 267$

48. Wang X, Hou Y, Li Q, Li X, Wang W, Ai X, Kuang T, Chen X, Zhang Y, Zhang J et al (2019) Rhodiola crenulata attenuates apoptosis and mitochondrial energy metabolism disorder in rats with hypobaric hypoxia-induced brain injury by regulating the HIF-1 $\alpha /$ microRNA 210/ISCU1/2(COX10) signaling pathway. J Ethnopharmacol 241:111801

49. Teipel SJ, Grothe M, Lista S, Toschi N, Garaci FG, Hampel H (2013) Relevance of magnetic resonance imaging for early detection and diagnosis of Alzheimer disease. Med Clin North Am 97(3):399-424

50. Suzuki H, Sumiyoshi A, Taki Y, Matsumoto Y, Fukumoto Y, Kawashima R, Shimokawa H (2013) Voxel-based morphometry and histological analysis for evaluating hippocampal damage in a rat model of cardiopulmonary resuscitation. Neuroimage $77: 215-221$

51. Ramírez-Ruiz B, Martí MJ, Tolosa E, Bartrés-Faz D, Summerfield C, Salgado-Pineda P, Gómez-Ansón B, Junqué C (2005) Longitudinal evaluation of cerebral morphological changes in Parkinson's disease with and without dementia. J Neurol 252(11):1345-1352

52. Niessen HG, Angenstein F, Vielhaber S, Frisch C, Kudin A, Elger CE, Heinze HJ, Scheich H, Kunz WS (2005) Volumetric magnetic resonance imaging of functionally relevant structural alterations in chronic epilepsy after pilocarpine-induced status epilepticus in rats. Epilepsia 46(7):1021-1026

53. Chen L, Cao S, Koehler RC, van Zijl PCM, Xu J (2020) Highsensitivity CEST mapping using a spatiotemporal correlationenhanced method. Magn Reson Med 84(6):3342-3350

54. Lee DH, Lee DW, Kwon JI, Woo CW, Kim ST, Lee JS, Choi CG, Kim KW, Kim JK, Woo DC (2019) In vivo mapping and quantification of creatine using chemical exchange saturation transfer imaging in rat models of epileptic seizure. Mol Imag Biol 21(2):232-239

55. Takahashi Y, Saito S, Kioka H, Araki R, Asano Y, Takashima S, Sakata Y, Yoshioka Y (2020) Mouse skeletal muscle creatine chemical exchange saturation transfer (CrCEST) imaging at 117T MRI. J Magn Reson Imaging JMRI 51(2):563-570

56. Zhou Z, Nguyen C, Chen Y, Shaw JL, Deng Z, Xie Y, Dawkins J, Marbán E, Li D (2017) Optimized CEST cardiovascular magnetic resonance for assessment of metabolic activity in the heart. J Cardiovasc Magn Reson Off J Soc Cardiovasc Magn Reson 19(1):95

57. Kogan F, Haris M, Debrosse C, Singh A, Nanga RP, Cai K, Hariharan H, Reddy R (2014) In vivo chemical exchange saturation transfer imaging of creatine (CrCEST) in skeletal muscle at 3T. J Magn Reson Imaging JMRI 40(3):596-602

58. Liso Navarro AA, Sikoglu EM, Heinze CR, Rogan RC, Russell VA, King JA, Moore CM (2014) Effect of diet on brain metabolites and behavior in spontaneously hypertensive rats. Behav Brain Res 270:240-247

59. Koundal S, Gandhi S, Kaur T, Khushu S (2014) Neurometabolic and structural alterations in rat brain due to acute hypobaric hypoxia: in vivo $1 \mathrm{H}$ MRS at 7 T. NMR Biomed 27(3):341-347 
60. Kreis R, Arcinue E, Ernst T, Shonk TK, Flores R, Ross BD (1996) Hypoxic encephalopathy after near-drowning studied by quantitative $1 \mathrm{H}$-magnetic resonance spectroscopy. J Clin Investig 97(5):1142-1154

61. Hwang J, DeLisi LE, Öngür D, Riley C, Zuo C, Shi X, Sung YH, Kondo D, Kim TS, Villafuerte R et al (2019) Cerebral bioenergetic differences measured by phosphorus-31 magnetic resonance spectroscopy between bipolar disorder and healthy subjects living in two different regions suggesting possible effects of altitude. Psychiatry Clin Neurosci 73(9):581-589

62. Fang B, Wang D, Huang M, Yu G, Li H (2010) Hypothesis on the relationship between the change in intracellular $\mathrm{pH}$ and incidence of sporadic Alzheimer's disease or vascular dementia. Int J Neurosci 120(9):591-595

63. Schwartz L, Peres S, Jolicoeur M, da Veiga MJ (2020) Cancer and Alzheimer's disease: intracellular $\mathrm{pH}$ scales the metabolic disorders. Biogerontology 21(6):683-694

64. Shi XF, Carlson PJ, Kim TS, Sung YH, Hellem TL, Fiedler KK, Kim SE, Glaeser B, Wang K, Zuo CS et al (2014) Effect of altitude on brain intracellular $\mathrm{pH}$ and inorganic phosphate levels. Psychiatry Res 222(3):149-156

65. Yao H, Zhao H, Wang J, Haddad GG (2018) Intracellular pH regulation in iPSCs-derived astrocytes from subjects with chronic mountain sickness. Neuroscience 375:25-33

66. Goldberg SV, Schoene RB, Haynor D, Trimble B, Swenson ER, Morrison JB, Banister EJ (1992) Brain tissue $\mathrm{pH}$ and ventilatory acclimatization to high altitude. J Appl Physiol (Bethesda, Md: 1985) 72(1):58-63

67. Abbott NJ, Friedman A (2012) Overview and introduction: the blood-brain barrier in health and disease. Epilepsia 53 Suppl 6(06): $1-6$

68. Saunders NR, Dziegielewska KM, Møllgård K, Habgood MD (2015) Markers for blood-brain barrier integrity: how appropriate is Evans blue in the twenty-first century and what are the alternatives? Front Neurosci 9:385

69. Tofts PS, Kermode AG (1991) Measurement of the blood-brain barrier permeability and leakage space using dynamic MR imaging. 1. Fundamental concepts. Magn Reson Med 17(2):357-367

70. Al-Bachari S, Naish JH, Parker GJM, Emsley HCA, Parkes LM (2020) Blood-brain barrier leakage is increased in Parkinson's disease. Front Physiol 11:593026

71. Joseph CR (2020) Novel MRI techniques identifying vascular leak and paravascular flow reduction in Early Alzheimer disease. Biomedicines 8(7):228

72. Al Ahmad A, Taboada CB, Gassmann M, Ogunshola OO (2011) Astrocytes and pericytes differentially modulate blood-brain barrier characteristics during development and hypoxic insult. J Cereb Blood Flow Metab Off J Int Soc Cereb Blood Flow Metab 31(2):693-705

73. Lafuente JV, Bermudez G, Camargo-Arce L, Bulnes S (2016) Blood-brain barrier changes in high altitude. CNS Neurol Disord: Drug Targets 15(9):1188-1197
74. Cramer NP, Korotcov A, Bosomtwi A, Xu X, Holman DR, Whiting K, Jones S, Hoy A, Dardzinski BJ, Galdzicki Z (2019) Neuronal and vascular deficits following chronic adaptation to high altitude. Exp Neurol 311:293-304

75. Jing L, Wu N, He L, Shao J, Ma H (2020) Establishment of an experimental rat model of high altitude cerebral edema by hypobaric hypoxia combined with temperature fluctuation. Brain Res Bull 165:253-262

76. Zlokovic BV (2008) The blood-brain barrier in health and chronic neurodegenerative disorders. Neuron 57(2):178-201

77. Montagne A, Barnes SR, Sweeney MD, Halliday MR, Sagare AP, Zhao Z, Toga AW, Jacobs RE, Liu CY, Amezcua L et al (2015) Blood-brain barrier breakdown in the aging human hippocampus. Neuron 85(2):296-302

78. Dheer A, Jain V, Kushwah N, Kumar R, Prasad D, Singh SB (2018) Temporal and spatial changes in glial cells during chronic hypobaric hypoxia: role in neurodegeneration. Neuroscience 383:235-246

79. Zhou Y, Huang X, Zhao T, Qiao M, Zhao X, Zhao M, Xu L, Zhao $\mathrm{Y}, \mathrm{Wu} \mathrm{L}, \mathrm{Wu} \mathrm{K}$ et al (2017) Hypoxia augments LPS-induced inflammation and triggers high altitude cerebral edema in mice. Brain Behav Immun 64:266-275

80. Carvey PM, Hendey B, Monahan AJ (2009) The blood-brain barrier in neurodegenerative disease: a rhetorical perspective. $\mathbf{J}$ Neurochem 111(2):291-314

81. Wang Y, Zhang R, Tao C, Xu Z, Chen W, Wang C, Song L, Zheng J, Gao F (2018) Blood-brain barrier disruption and perivascular beta-amyloid accumulation in the brain of aged rats with spontaneous hypertension: evaluation with dynamic contrast-enhanced magnetic resonance imaging. Korean J Radiol 19(3):498-507

82. Duarte Lobo D, Nobre RJ, Oliveira Miranda C, Pereira D, Castelhano J, Sereno J, Koeppen A, Castelo-Branco M, Pereira de Almeida L (2020) The blood-brain barrier is disrupted in Machado-Joseph disease/spinocerebellar ataxia type 3: evidence from transgenic mice and human post-mortem samples. Acta Neuropathol Commun 8(1):152

83. Godbout JP, Johnson RW (2009) Age and neuroinflammation: a lifetime of psychoneuroimmune consequences. Immunol Allergy Clin North Am 29(2):321-337

84. Makaryus R, Lee H, Yu M, Zhang S, Smith SD, Rebecchi M, Glass PS, Benveniste H (2011) The metabolomic profile during isoflurane anesthesia differs from propofol anesthesia in the live rodent brain. J Cereb Blood Flow Metab Off J Int Soc Cereb Blood Flow Metab 31(6): 1432-1442

85. Rzemieniec J, Litwa E, Wnuk A, Lason W, Krzeptowski W, Kajta M (2016) Selective aryl hydrocarbon receptor modulator 3,3'-diindolylmethane impairs AhR and ARNT signaling and protects mouse neuronal cells against hypoxia. Mol Neurobiol 53(8):5591-5606

Publisher's Note Springer Nature remains neutral with regard to jurisdictional claims in published maps and institutional affiliations. 